\title{
Triad Resonance between Gravity and Vorticity Waves in Vertical Shear
}

\author{
Theodore D. Drivas ${ }^{a, b 1}$ and Scott Wunsch ${ }^{a}$ \\ ${ }^{a}$ The Johns Hopkins Applied Physics Laboratory \\ ${ }^{b}$ Department of Applied Mathematics $\mathcal{E}$ Statistics \\ The Johns Hopkins University
}

\begin{abstract}
Weakly nonlinear theory is used to explore the effect of vertical shear on surface gravity waves in three dimensions. An idealized piecewise-linear shear profile motivated by wind-driven profiles and ambient currents in the ocean is used. It is shown that shear may mediate weakly nonlinear resonant triad interactions between gravity and vorticity waves. The triad results in energy exchange between gravity waves of comparable wavelengths propagating in different directions. For realistic ocean shears, shear-mediated energy exchange may occur on timescales of minutes for shorter wavelengths, but slows as the wavelength increases. Hence this triad mechanism may contribute to the larger angular spreading (relative to wind direction) for shorter wind-waves observed in the oceans.
\end{abstract}

Keywords: Weakly Nonlinear Waves, Wave-Current Interaction, Triad Resonance

\section{Introduction}

The interaction of surface gravity waves with non-uniform mean currents is ubiquitous in oceanography [1]. Both wind-driven (transient) and permanent currents, such as the Gulf Stream and Kuroshio, may interact with gravity waves. Typical wind-driven currents have speeds of the order of tens of centimeters per second, while permanent currents may approach a few meters per second. Near-surface vertical shears may be as large as .02 $\mathrm{s}^{-1}$ [2].

The presence of a mean subsurface shear can alter the standard dispersion relations, wave lengths and velocity profiles of the propagating gravity waves [3], and may facilitate the nonlinear transfer of energy among waves [4]. Mean shears leading to nonlinear steepening are also among the many proposed mechanisms for the appearance of rogue waves - relatively large waves compared to the average wave height in the vicinity, sometimes capable of destroying ocean vessels [5]. Currents and shear may also play a role in the development of the observed directional spectrum of wind-waves in the ocean. In particular, wavelengths shorter than the spectral peak typically exhibit a much broader directionality (relative to the wind direction), while longer waves are more closely aligned with the wind direction $[6,7,8]$. Here, nonlinear interactions involving shear will be considered as a possible mechanism for the directional spreading of short wavelength gravity waves.

Many theoretical approaches have been applied to analyze the effect of currents and shear on gravity waves. Longuet-Higgins and Stewart [9] considered the exchange of energy between a current with slow horizontal variations and a linear gravity wave. Craik [10] considered waves in a uniform shear flow and found that a resonant triad exists due to the presence of a critical level (a depth where the current matches the wave phase velocity) in uniform shear. This is of interest because three-wave interactions are not possible in the absence of a mean current; the lowest order wave interaction involves four gravity waves $[11,12,13]$. However, most realistic ocean currents are slower than gravity wave phase speeds (a few meters per second), prohibiting the critical level triad from acting in the oceans. The present work focuses on currents that are too slow to allow critical levels.

Most gravity wave studies rely on the assumption of potential flow. Vertically varying shear, with its inherent vorticity, is incompatible with this assumption [14]. Several studies have relaxed the irrotational condition to allow vorticity in the underlying mean flow. Most of these have been restricted to two dimensions, where the stream function

\footnotetext{
${ }^{1}$ Corresponding author email: tdrivas $2 @$ jhu.edu
} 
is used to formulate the problem in a manner very similar to potential flow $[15,16,18]$. In three dimensions, McHugh [19] used an approximate linearized formulation to investigate surface waves propagating over shear in a channel, while Nwogu [20] utilized a general formulation of the 3D non-potential shear-surface interaction for numerical solution.

A more general analytic approach to vertical shear and gravity waves was developed by Zakharov and Shrira using a Taylor expansion of the boundary condition about its mean position [21]. The vertical velocity profile obeys the Rayleigh equation [22] in this formulation. The method of Zakharov and Shrira [21] is used here as well. However, the previous work focused on wave interactions with critical levels, while waves with phase speeds which are too fast for critical levels to exist are considered here. Shrira [23] utilized the linear equations of motion to develop solutions of the Rayleigh equation for arbitrary vertical shear profiles and showed that shear may alter the dispersion relation of gravity waves.

Here, previously developed analytic methods for gravity waves in vertical shear $[21,23]$ are applied in three dimensions. Current speeds are restricted to be slower than the gravity wave phase speeds, so that critical levels do not arise. This restriction is realistic for most ocean environments. Previous linear solutions of the Rayleigh equation for a piece-wise linear current with a free surface are extended to three dimensions. There are three permitted modes, two surface gravity waves (slightly modified by the shear) and one vorticity wave. This vorticity wave, sometimes also called an edge wave or Rayleigh wave, is introduced by the curvature of the mean profile which, in this case, is a delta function located at the base of the shear layer. The vorticity wave is localized in a neighborhood of that depth. For larger shears, one can have instability $[24,25]$ but for small values the unstable region becomes vanishingly small.

The focus of the present analysis is on resonant triads. These are combinations of waves with wavenumbers $\mathbf{k}, \mathbf{p}, \mathbf{q}$ and frequencies $\omega_{\mathbf{k}}, \omega_{\mathbf{p}}, \omega_{\mathbf{q}}$ satisfying

$$
\begin{aligned}
\mathbf{k} & =\mathbf{p}+\mathbf{q} \\
\omega_{\mathbf{k}} & =\omega_{\mathbf{p}}+\omega_{\mathbf{q}}
\end{aligned}
$$

Two of the waves are surface gravity waves with comparable frequencies and wavelengths but propagating in different directions. The third will be a vorticity wave solution of the Rayleigh equation, and can mediate the transfer of energy between the two gravity waves. This is analogous to triads in two-layer fluids, in which an interfacial wave on the pycnocline mediates the transfer of energy between a pair of gravity waves on the surface [26, 27, 28].

It has been conjectured that such triads involving an interfacial wave contribute to energy transfer among gravity waves in the ocean [29]. Wave triads are lower order interactions and hence potentially stronger than the four-wave couplings allowed in the absence of interfacial or vorticity waves $[11,12,13]$. Here, weakly nonlinear analysis is used to estimate the rate of energy transfer for triads involving two gravity waves and a vorticity wave. The potential for such transfers to play a role in the development of observed wind-wave spectra $[6,7,8]$ is evaluated. Zakharov and Shrira [21] also proposed that the interaction with shear could be a possible mechanism for the spreading of the angular spectrum of wind waves, but postulated critical levels as the dominant energy exchange mechanism. This restricts the validity to gravity waves with phase speeds which are slower than the mean current, which is typically a small segment of the complete spectrum. The present work focuses on faster waves, and hence investigates an alternate mechanism of energy transfer which may be applicable to a broader segment of the wave spectrum.

\section{Problem Formulation}

The interaction of gravity waves with vertical shear is investigated here by assuming a homogeneous, incompressible ocean of infinite extent and depth. A stationary horizontal current with amplitude depending only on depth is assumed, and wave-like perturbations to the free surface are studied. Wind is not explicitly considered, but in oceanographic applications would often be the driving force for the current. Surface tension and rotation are also neglected. Equations for weakly nonlinear interactions between gravity waves in the presence of vertical shear are derived, as in $[21,23]$. To obtain quantitative results, an idealized current profile is assumed later in the analysis, as in [24, 25]. 


\subsection{Equations of Motion}

Let $\mathbf{x}=(x, y, z)$ where the $z$ coordinate runs vertically. Let $\mathbf{U}(z)=\left(U_{1}(z), U_{2}(z), 0\right)$ be the stationary (in time) horizontal shear flow beneath the mean free surface $(z=0)$. To simplify the problem, $\mathbf{U}$ is a function only of depth $z$; horizontal variations of the current are not considered. Notice that by construction $\mathbf{U}$ is a divergence free vector field and hence satisfies the continuity equation. Let $P(\mathbf{x}, t)$ be the pressure required to maintain the velocity field $\mathbf{U}$ and $\rho$ be the constant mass density of the fluid. The pair $(\mathbf{U}, P)$ satisfies the incompressible Euler equation subject to gravity.

Perturbations to the flow are described by the velocity and pressure fluctuations $\mathbf{u}(\mathbf{x}, t)$ and $p(\mathbf{x}, t)$. These may deform the free surface height by $\eta(x, y, t)$ from its mean position $z=0$. The full velocity field is given by $\mathbf{U}(z)+\mathbf{u}(\mathbf{x}, t)$ where $\mathbf{u}=\left(u_{1}, u_{2}, u_{3}\right)$ and the full pressure field by $P(\mathbf{x}, t)+p(\mathbf{x}, t)$.

Figure 1 illustrates the components of the perturbed fluid motion. The velocity and pressure perturbations satisfy the momentum and continuity equations:

$$
\begin{aligned}
& D_{t} \mathbf{u}(\mathbf{x}, t)+\mathbf{u} \cdot \nabla \mathbf{u}+u_{3}(\mathbf{x}, t) \mathbf{U}^{\prime}(z)=-\nabla p(\mathbf{x}, t) / \rho \\
& \nabla \cdot \mathbf{u}(\mathbf{x}, t)=0
\end{aligned}
$$

Here $D_{t} \equiv \partial_{t}+\mathbf{U}(z) \cdot \nabla_{\mathrm{H}}$ is the convective derivative, $\nabla_{\mathrm{H}} \equiv\left(\partial_{x}, \partial_{y}, 0\right)$ is the horizontal gradient, and $\mathbf{U}^{\prime}(z)$ is the vertical shear vector $\left(U_{1}^{\prime}(z), U_{2}^{\prime}(z), 0\right)$. Using the fact that the velocity field is incompressible, the pressure term may be eliminated (at the expense of adding higher-order derivatives) by straightforward calculation [21]:

$$
\begin{aligned}
& D_{t}\left(\partial_{z}^{2}+\Delta_{\mathrm{H}}\right) u_{3}-\mathbf{U}^{\prime \prime}(z) \cdot \nabla_{\mathrm{H}} u_{3}=\mathcal{N}_{3}, \\
& D_{t} \Delta_{\mathrm{H}} u_{i}+\left(U_{i}^{\prime} \Delta_{\mathrm{H}}+D_{t} \partial_{z} \partial_{i}-\mathbf{U}^{\prime} \cdot \nabla_{\mathrm{H}} \partial_{i}\right) u_{3}=\mathcal{N}_{i},
\end{aligned}
$$

where $i=1,2, \Delta_{\mathrm{H}} \equiv \nabla_{\mathrm{H}}^{2}=\partial_{x}^{2}+\partial_{y}^{2}$ is the horizontal Laplacian and the nonlinear terms $\mathcal{N}_{i}$ for $j=1,2,3$ defined as

$$
\mathcal{N}_{j} \equiv \partial_{j} \nabla_{\mathrm{H}} \cdot[\mathbf{u} \cdot \nabla \mathbf{u}]-\Delta_{\mathrm{H}}\left[\mathbf{u} \cdot \nabla u_{j}\right]
$$

No assumption regarding the nature or magnitude of the perturbation $\mathbf{u}$ has been made in deriving the above equations. Equations (5) and (6), along with appropriate boundary conditions, govern the dynamics of the perturbations. Section 2.2 discusses the free surface boundary condition. The linearization of Equation (5) (setting $\mathcal{N}_{3}=0$ ) is known as the Rayleigh equation, which is the inviscid limit of the Orr-Sommerfeld equation [30]. It governs perturbations to shear flow quite generally [22]

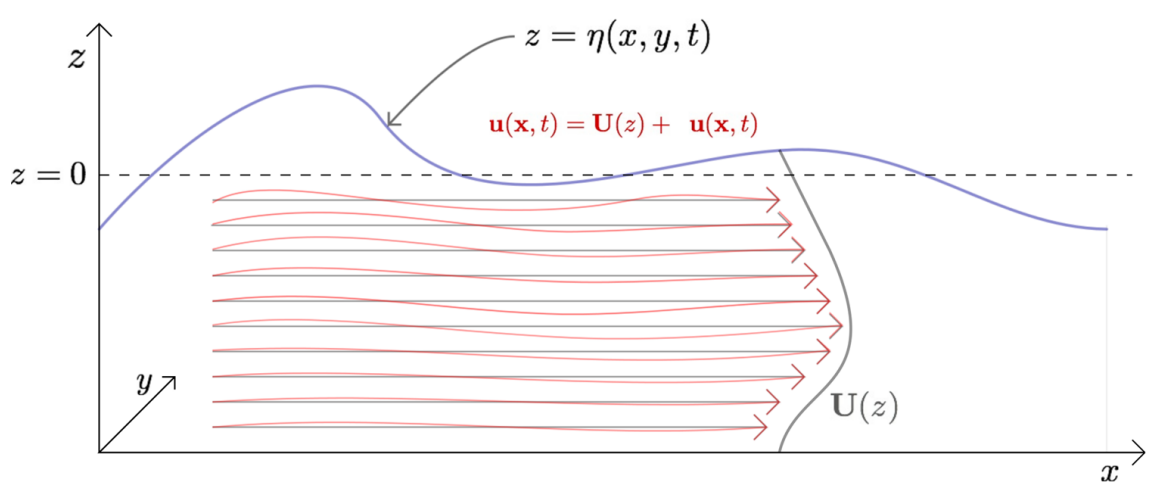

Figure 1: Depicted is a shear flow perturbed by the action of a surface wave. The deformed red arrows superimposed on the underlying grey arrows represent the effect of this perturbation to the mean shear flow. The free surface is now mobile and its contortions are due to the vertical motion of the fluid below. 
which, for the assumed plane wave (Eq. 13), reduces to

$$
g|\mathbf{k}|^{2} v_{3}=(\omega-\mathbf{k} \cdot \mathbf{U})^{2} \partial_{z} v_{3}+(\omega-\mathbf{k} \cdot \mathbf{U}) \mathbf{k} \cdot \mathbf{U}^{\prime} v_{3}
$$

with the nonlinear terms defined by:

\section{Linear Dispersion and Resonant Triads} distance $h$ :

$$
\mathbf{U}(z)=\left\{\begin{array}{lr}
\mathbf{U}_{0}\left(1+\frac{z}{h}\right) & -h \leq z \leq 0 \\
0 & z<-h
\end{array}\right.
$$
relation, a plane wave solution is assumed (for $j=1,2,3$ ):

$$
u_{j}(\mathbf{x}, z, t)=\Re e v_{j}(z) \exp (i \mathbf{k} \cdot \mathbf{x}-i \omega t) .
$$

Substituting Equation 13 into Equation 5 and keeping only the linear terms $(\mathcal{N}=0)$ yields

$$
(\omega-\mathbf{k} \cdot \mathbf{U})\left(\partial_{z}^{2}-|\mathbf{k}|^{2}\right) v_{3}-\mathbf{k} \cdot \mathbf{U}^{\prime \prime}(z) v_{3}=0 .
$$

The linearized free surface boundary condition (read off from Eq. (10)) is

$$
\left(D_{t}^{2} \partial_{z}-\mathbf{U}^{\prime} \nabla_{\mathrm{H}} D_{t}-g \Delta_{H}\right) u_{3}=0
$$

$$
\begin{aligned}
\mathcal{N}(\mathbf{u}, \eta) \equiv & \eta \mathbf{U}^{\prime} \cdot \nabla_{\mathrm{H}}\left(D_{t} \partial_{z} u_{3}-\mathbf{U}^{\prime} \cdot \nabla u_{3}\right)+\nabla_{\mathrm{H}} \cdot D_{t}[\mathbf{u} \cdot \nabla \mathbf{u}]+\eta D_{t}^{2} \Delta_{\mathrm{H}} u_{3}+D_{t} \eta D_{t} \Delta_{\mathrm{H}} u_{3}+2 D_{t} \nabla_{\mathrm{H}} \eta \cdot D_{t} \nabla_{\mathrm{H}} u_{3} \\
& +2 \nabla_{\mathrm{H}} \eta \cdot D_{t}^{2} \nabla_{\mathrm{H}} u_{3}+D_{t} \Delta_{\mathrm{H}} \eta D_{t} u_{3}+\Delta_{\mathrm{H}} \eta D_{t}^{2} u_{3}+g \eta \Delta_{\mathrm{H}} \partial_{z} u_{3}+2 g \nabla_{\mathrm{H}} \eta \cdot \nabla_{\mathrm{H}} \partial_{z} u_{3}+g \partial_{z} u_{3} \Delta_{\mathrm{H}} \eta \\
& -g 2 \partial_{\ell} \delta u_{j} \partial_{\ell} \partial_{j} \eta-g \mathbf{u} \cdot \nabla_{\mathrm{H}}\left(\Delta_{\mathrm{H}} \eta\right)-g\left(\Delta_{\mathrm{H}} \mathbf{u}\right) \cdot \nabla_{\mathrm{H}} \eta
\end{aligned}
$$

where indices $j$ and $\ell$ sum only over the horizontal coordinates 1,2 and all terms are evaluated at $z=0$. Details of the boundary condition derivation are found in Appendix A. This result is identical to that found in [21] to second order.

To investigate the phenomenology of wave interactions with vertical shear, a piece-wise linear background current profile is used here. The assumed profile consists of a surface current $\mathbf{U}_{0}$ which decreases linearly with depth over a

This piece-wise linear current has been previously investigated in two dimensions by [24, 25]. To find the dispersion

\footnotetext{
${ }^{2}$ We note that, to linear order, Equations (3) combined with the surface boundary conditions show that the total energy equation for the perturbation is $\frac{\mathrm{d}}{\mathrm{d} t} \int \mathrm{d} \mathbf{x}_{H} \frac{1}{2}\left(\int|\mathbf{u}|^{2} \mathrm{~d} z+g \eta^{2}\right)=-\int \mathrm{d} \mathbf{x}_{H} \int \mathrm{d} z\left(\mathbf{u}_{H} \cdot \mathbf{U}^{\prime}\right) u_{3}$ where $\mathbf{x}_{H}=(x, y)$ and $\mathbf{u}_{H}=\left(u_{1}, u_{2}\right)$.
} 
evaluated at the mean free surface $(z=0)$. In two dimensions, the linear problem (Eq. 14 and 16) reduces to that investigated by Shrira [23], and specifically for the background profile of Equation 12, by Bakas et al.[25] and Voronovich et al.[24].

The solution is found by means of matching conditions at the velocity "kink" located at $z=-h$. This approach is based on [30] and has been used previously by others [24, 25, 33] to find two-dimensional linear dispersion relations in piece-wise linear profiles. The details are found in Appendix B. Since $\mathbf{U}^{\prime \prime}=0$ everywhere except at this depth, the solution is straightforward and critical level effects do not arise. The solution is

$$
v_{3}(z)=\left\{\begin{array}{lr}
e^{|\mathbf{k}| z}+\mathcal{B} e^{-|\mathbf{k}| z+2 h)} & -h \leq z \leq 0 \\
C e^{|\mathbf{k}| z} & z \leq-h
\end{array}\right.
$$

$$
\mathcal{B} \equiv \frac{\mathbf{k} \cdot \mathbf{U}_{0}}{2 \omega a-\mathbf{k} \cdot \mathbf{U}_{0}}, \quad C \equiv \frac{2 \omega a}{2 \omega a-\mathbf{k} \cdot \mathbf{U}_{0}}
$$

where $a \equiv|\mathbf{k}| h$ is the normalized wavenumber. The instance of a vanishing denominator (which will happen for a fixed current profile for specific wavenumber), results in $e^{\mathbf{k} \mid z}$ for $-h \leq z \leq 0$ in formula (17) vanishing. Substituting into the free surface boundary condition (Eq. 16) yields the cubic dispersion relation:

$$
\begin{aligned}
\left(\omega-\mathbf{k} \cdot \mathbf{U}_{0}\right)^{3} & +C_{2}\left(\omega-\mathbf{k} \cdot \mathbf{U}_{0}\right)^{2}+C_{1}\left(\omega-\mathbf{k} \cdot \mathbf{U}_{0}\right)+C_{0}=0 \\
\text { where } \quad C_{0} & =\frac{g|\mathbf{k}| \mathbf{k} \cdot \mathbf{U}_{0}}{2 a}\left(1-2 a-e^{-2 a}\right) \\
C_{1} & =-\frac{\left(\mathbf{k} \cdot \mathbf{U}_{0}\right)^{2}}{2 a^{2}}\left(1-2 a-e^{-2 a}\right)-g|\mathbf{k}| \\
C_{2} & =\frac{\mathbf{k} \cdot \mathbf{U}_{0}}{2 a}\left(1+2 a-e^{-2 a}\right)
\end{aligned}
$$

The dispersion relation (Eq. (19)) is cubic and therefore has three (possibly complex) roots. In the absence of shear, it reduces to the standard surface gravity wave dispersion relation ${ }^{3},\left(\omega-\mathbf{k} \cdot \mathbf{U}_{0}\right)^{2}=g|\mathbf{k}|$. In general, two roots of Equation 19 correspond to gravity wave modes which would exist in the absence of the kink in the background velocity profile. The third root is a "vorticity" wave, "edge" wave or Rayleigh wave [22], which travels along the kink and would exist even if the surface were a rigid lid. Figure 2 shows the vertical dependence Eq. (17) In two dimensions, this dispersion relation reduces to one of the cases studied by Bakas and Ioannou [25]. For a strong current $\left(\mathbf{k} \cdot \mathbf{U}_{0}\right)^{2} \gg g|\mathbf{k}|$, the interaction of the surface and vorticity waves result in an instability. More generally, velocity profiles with 2 kinks permit a wider range of instabilities due to the interaction of two vorticity waves [22]. This has been dubbed the "explosive instability" [24] and studied in two dimensions by [25].

A key prediction of the piecewise linear model is the appearance of the third mode, refered to as a "vorticity wave" or "edge wave." For resonant triads involving vorticity waves to occur in the ocean, this mode must exist for more realistic current profiles. Although Shrira and Sazonov [31] argued that piece-wise linear profiles have qualitatively different dispersion relations than continuous profiles, more recent work has shown that their use produces realistic results. For example, Bakas and Ioannou [25] show that, in two dimensions, numerical solutions of the Rayleigh equation with smoothed profiles have approximately the same dispersion relation as the kinked linear profiles used here. They also show that phase locking with the vorticity wave produces a normal mode and the structure of this normal mode remains roughly the same for both kinked and smoothed profiles. Also, Chen and Walsh [32] provide a rigorous justification of the use of piecewise linear density profiles for studying stratified steady water waves. A kinked velocity profile with a density interface (mathematically analogous to a free surface) has also been shown numerically to have nearly the same dispersion relation and structure function as a smoothed profile [33]. Plougonven et al. [42] show by means of asymptotic expansions that for a smoothed profile a similar structure as the "ideal" edge

\footnotetext{
${ }^{3}$ Taking the limit of Eq. (19) directly yields $\omega\left(\left(\omega-\mathbf{k} \cdot \mathbf{U}_{0}\right)^{2}-g|\mathbf{k}|\right)=0$, which admits the two correct limiting frequencies and a spurious trivial frequency. This third mode corresponds to a vertical profile (17) which has an unphysical peak at the kink at "minus infinity". Formally, taking the limit $h \rightarrow \infty$ in Eq. (14), forces Eq. (17) to reduce to $v_{3}(z)=e^{|\mathbf{k}| z}$. Upon substitution into Eq. 16 yields the correct algebraic relation.
} 
(a)

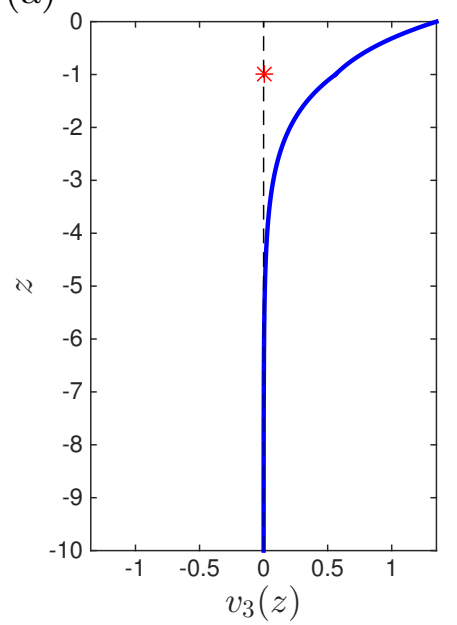

(b)

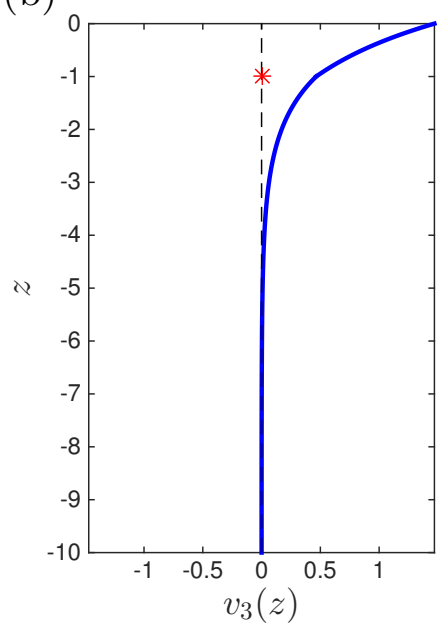

(c)



Figure 2: Plotted are normalized eigenfunctions $v_{3}(z)$ with depth. The kink is located at a depth of $h=1 \mathrm{~m}$ (indicated by the asterisk (*) and the wavenumber is fixed at $|\mathbf{k}|=1$. The subplots $(a)$ and $(b)$ depict the depth dependence for the two gravity modes and the subplot $(c)$, the shear mode.

wave exists. These results support the expectation that no anomalies are introduced by the use of the kinked profile in the present work.

Here we focus on weak currents, for which the three roots of Equation 19 are all real and hence stable, unlike the work of Bakas and Ioannou [25]. For small shear, the dispersion relation has the approximate form

$$
\begin{aligned}
\omega-\mathbf{k} \cdot \mathbf{U}_{0} \simeq \pm \sqrt{g|\mathbf{k}|}-\frac{\mathbf{k} \cdot \mathbf{U}_{0}}{2 a}\left(1-e^{-2 a}\right) & & \text { (gravity waves) } \\
\omega-\mathbf{k} \cdot \mathbf{U}_{0} \simeq \frac{\mathbf{k} \cdot \mathbf{U}_{0}}{2 a}\left(1-2 a-e^{-2 a}\right) & & \text { (vorticity wave) }
\end{aligned}
$$

in the limit $\frac{\mathbf{k} \cdot \mathbf{U}_{0}}{2 a} \ll \sqrt{g|\mathbf{k}|}$ (small Froude number). The presence of a small shear linearly shifts the gravity wave dispersion relation and makes the vorticity wave possible. For realistic ocean currents with speeds of order tens of centimeters and depths of order tens of meters, $\frac{\mathbf{k} \cdot \mathbf{U}_{0}}{2 a} \simeq 10^{-4}-10^{-1} \mathrm{sec}^{-1}$, which is much smaller than the intrinsic gravity wave frequency $\sqrt{g|\mathbf{k}|}$ for all but very long ( $\gg 100$ meter) gravity waves. Hence this approximation will typically be applicable.

The lowest order interaction of waves is a triad resonance, in which a combination of waves satisfies Equations 1 and 2. It is well known that, in the absence of shear, the gravity wave dispersion relation does not allow any triad solutions, and the lowest order interaction is a quartic (4 wave) resonance $[11,12,13]$. The shear-modified dispersion relation makes triad resonance possible, with two gravity waves and one vorticity wave interacting, as first noticed by Voronovich [24]. This is analogous to triad resonance in stratified two-layer fluids, in which an interfacial wave interacts with two gravity waves [26, 27, 28].

The wavenumbers involved in the triad interaction are depicted in Figure 3. In this analysis, the gravity waves are assigned wavenumbers $\mathbf{k}$ and $\mathbf{p}$, which form angles $\vartheta_{\mathbf{k}}$ and $\vartheta_{\mathbf{p}}$ with the background current $\mathbf{U}_{0}$. The vorticity wave has wavenumber $\mathbf{q}$ and angle $\vartheta_{\mathbf{q}}$. Given the two gravity waves, the vorticity wavenumber must satisfy

$$
|\mathbf{q}|^{2}=|\mathbf{k}|^{2}+|\mathbf{p}|^{2}-2|\mathbf{k}| \mathbf{p} \mid \cos \left(\vartheta_{\mathbf{k}}-\vartheta_{\mathbf{p}}\right) .
$$

$$
\vartheta_{\mathbf{q}}=\cos ^{-1}\left(\frac{|\mathbf{k}| \cos \left(\vartheta_{\mathbf{k}}\right)-|\mathbf{p}| \cos \left(\vartheta_{\mathbf{p}}\right)}{|\mathbf{q}|}\right)
$$

Using these, the frequency triad condition may be expressed as

$$
\frac{2 h}{\left|\mathbf{U}_{0}\right|}(\sqrt{g|\mathbf{k}|}-\sqrt{g|\mathbf{p}|})=\cos \vartheta_{\mathbf{k}}\left(1+2|\mathbf{k}| h-e^{-2|\mathbf{k}| h}\right)-\cos \vartheta_{\mathbf{p}}\left(1+2|\mathbf{p}| h-e^{-2|\mathbf{p}| h}\right)-\cos \vartheta_{\mathbf{q}}\left(1-e^{-2|\mathbf{q}| h}\right)
$$




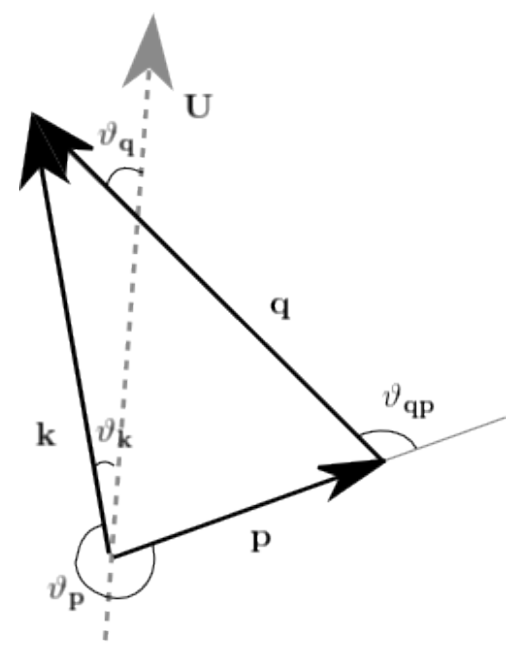

Figure 3: Depicted is a triad $\mathbf{k}=\mathbf{p}+\mathbf{q}$ for $\mathbf{k}$, the primary gravity wave at angle $\vartheta_{\mathbf{k}}$ with the current $\mathbf{U}$.

The resonant triad condition is depicted graphically in Figure 4 in the quasi-2D case where the waves are aligned/antialigned with the current, i.e. $\mathbf{k} \cdot \mathbf{U}= \pm|\mathbf{k} \| \mathbf{U}|$. In two-dimensions (one horizontal, one vertical), pick a point $(\omega(\mathbf{k}), \mathbf{k})$ on the dispersion curve corresponding to one of the three solutions of Equation 19 (solid lines in Figure 4, colors correspond to different roots). Now reproduce all branches of the dispersion relation, shifting the origin to be at $(\omega(\mathbf{k}), \mathbf{k})$, drawn as dashed lines in Figure 4. Then any other point where the dashed curves intersect a solid one will have some coordinates $(\omega(\mathbf{p}), \mathbf{p})$ in the original frame and $(\omega(\mathbf{q}), \mathbf{q})$ in the translated one and so by construction the resonant triad condition (Eq. 1 and 2) is satisfied. Without the shear mode there can be no non-trivial intersection of these curves for 2D waves. Generically that there will be multiple resonant triad pairs from the interaction between gravity and vorticity waves. This is reminiscent of gravity wave - internal wave interaction (e.g. [28]). Technically the presentation just outlined is for the two dimensional case; it works more generally in three dimensions but intersections occur between two dimensional surfaces rather than simply lines and a richer class of triad interactions are possible.

The triad condition and dispersion relation generally force the vorticity wave to have a much smaller frequency than the gravity waves. Hence an approximate triad solution may be found by assuming the wavenumber amplitude (and hence also frequency) of the second gravity wave differs from the first by a small amount $\delta$, defined by $|\mathbf{p}|=$ $|\mathbf{k}|(1-\delta)$. Then, to lowest order, the vorticity wave has

$$
|\mathbf{q}| \simeq 2|\mathbf{k}|\left|\sin \frac{1}{2}\left(\vartheta_{\mathbf{k}}-\vartheta_{\mathbf{p}}\right)\right| .
$$

$$
\cos \vartheta_{\mathbf{q}} \simeq \frac{\cos \vartheta_{\mathbf{k}}-\cos \vartheta_{\mathbf{p}}}{\left.2 \sin \frac{1}{2} \mid \vartheta_{\mathbf{k}}-\vartheta_{\mathbf{p}}\right) \mid}
$$

Given these, the triad condition (Eq. 27) may be solved for $\delta$ to lowest order as a function of $\vartheta_{\mathbf{k}}$ and $\vartheta_{\mathbf{p}}$ :

$$
\delta=\frac{|\mathbf{k}|\left|\mathbf{U}_{0}\right|}{\sqrt{g|\mathbf{k}|}} \frac{\cos \vartheta_{\mathbf{k}}-\cos \vartheta_{\mathbf{p}}}{a}\left(1+2 a-e^{-2 a}-\frac{1-e^{-4 a \sin \frac{1}{2}\left|\vartheta_{\mathbf{k}}-\vartheta_{\mathbf{p}}\right|}}{2 \sin \frac{1}{2}\left|\vartheta_{\mathbf{k}}-\vartheta_{\mathbf{p}}\right|}\right)
$$

where $a \equiv|\mathbf{k}| h$. Notice that for $\vartheta_{\mathbf{k}}=\vartheta_{\mathbf{p}}$, Equation 30 reduces to $\delta=0$ (and therefore $\mathbf{k}=\mathbf{p}$ ), as expected. The dimensionless change $\delta$ to the wavenumber amplitude is of the order of $\frac{|\mathbf{k}| \mathbf{U}_{0} \mid}{\sqrt{g \mid \mathbf{k}} \mid}$, which for gravity waves with wavelengths of order 10 meters and surface currents of order 10 centimeters is of the order of $0.01-0.1$. Hence the approximation that $|\mathbf{k}| \simeq|\mathbf{p}|$ is valid, and improves as the wavelength increases ( $|\mathbf{k}|$ decreases) or the current magnitude decreases.

Figure 5 presents the angular dependence of $\delta$ (Eq. 30) for $a=10$ and several values of the angle $\vartheta_{\mathbf{k}}$ between the current and the first gravity wave (wavenumber $\mathbf{k}$ ). The functional form is nearly independent of $a$ for $0.1 \leq a \leq 100$, 
$\omega(\mathrm{k})$

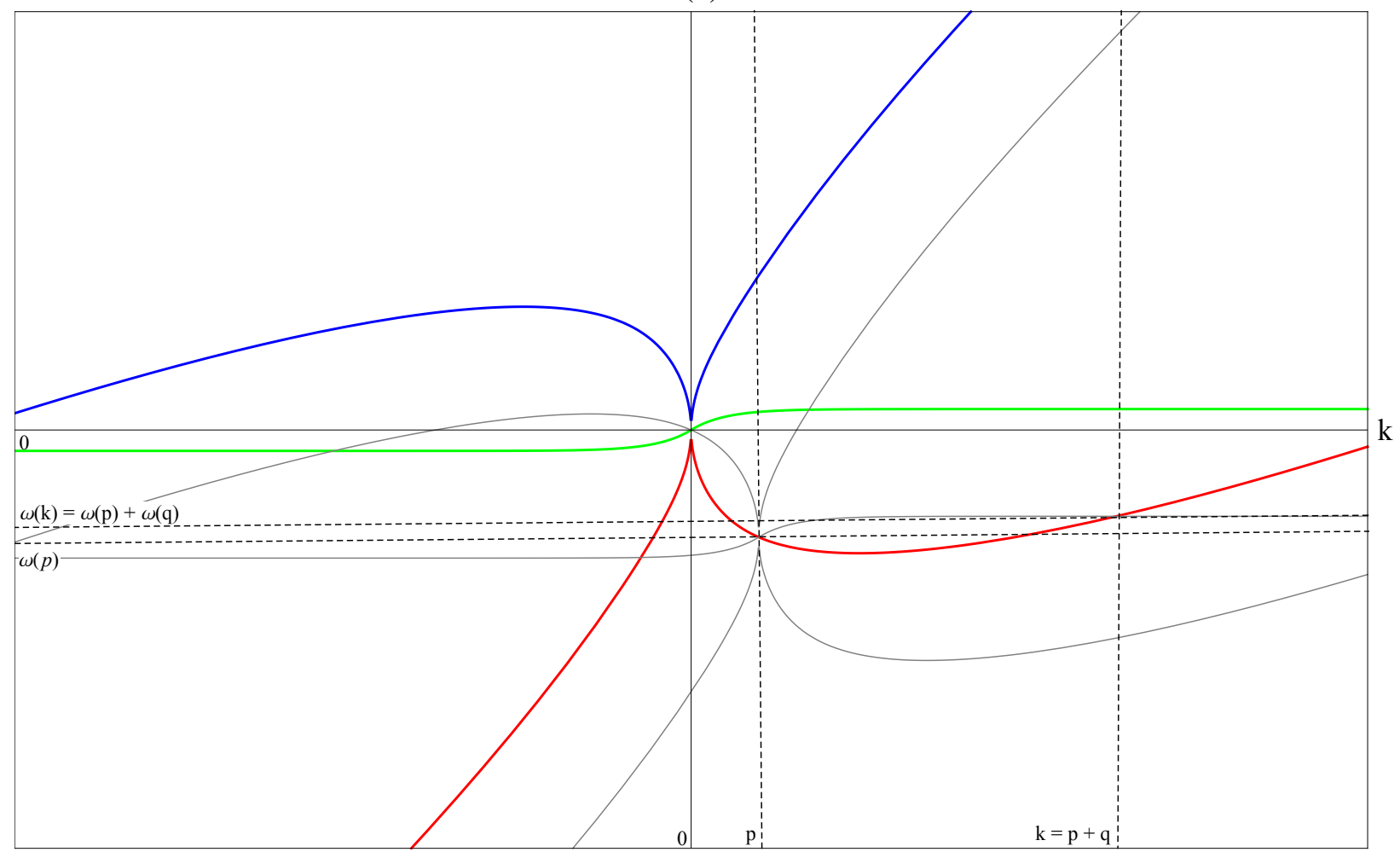

Figure 4: The three branches of the dispersion curve for waves over a piecewise linear profile are shown for co-propagating and counter propagating waves to the current (i.e. the situation is quasi-2D with $\mathbf{k} \cdot \mathbf{U}= \pm|\mathbf{k} \| \mathbf{U}|$ ). The red and blue modes are the Doppler shifted surface waves (with dispersion relations modified by shear) while the green shear mode appears only in the presence of subsurface shear. The gray curves are shifted version of these dispersion curves. The dashed vertical and horizontal lines serve as an aid to highlight intersections between the gray shifted dispersion curves and the primary colored curves which represent resonant triads.

which would be typical of oceanographic conditions. For small shear, the resonant triad connects one gravity wave with another of similar wavelength but with a significantly different direction. If $\mathbf{k}$ represents the direction of initial energy input (due to the wind, for example), Figure 5 suggests that near-surface shear can mediate the spreading of gravity wave energy into directions other than the direction of initial energy input. This could result in the spreading of the directional wave spectrum if the timescale of energy transfer is rapid enough. In the next section, a weakly nonlinear analysis is used to estimate the energy transfer rate as a function of wavelength and direction. 


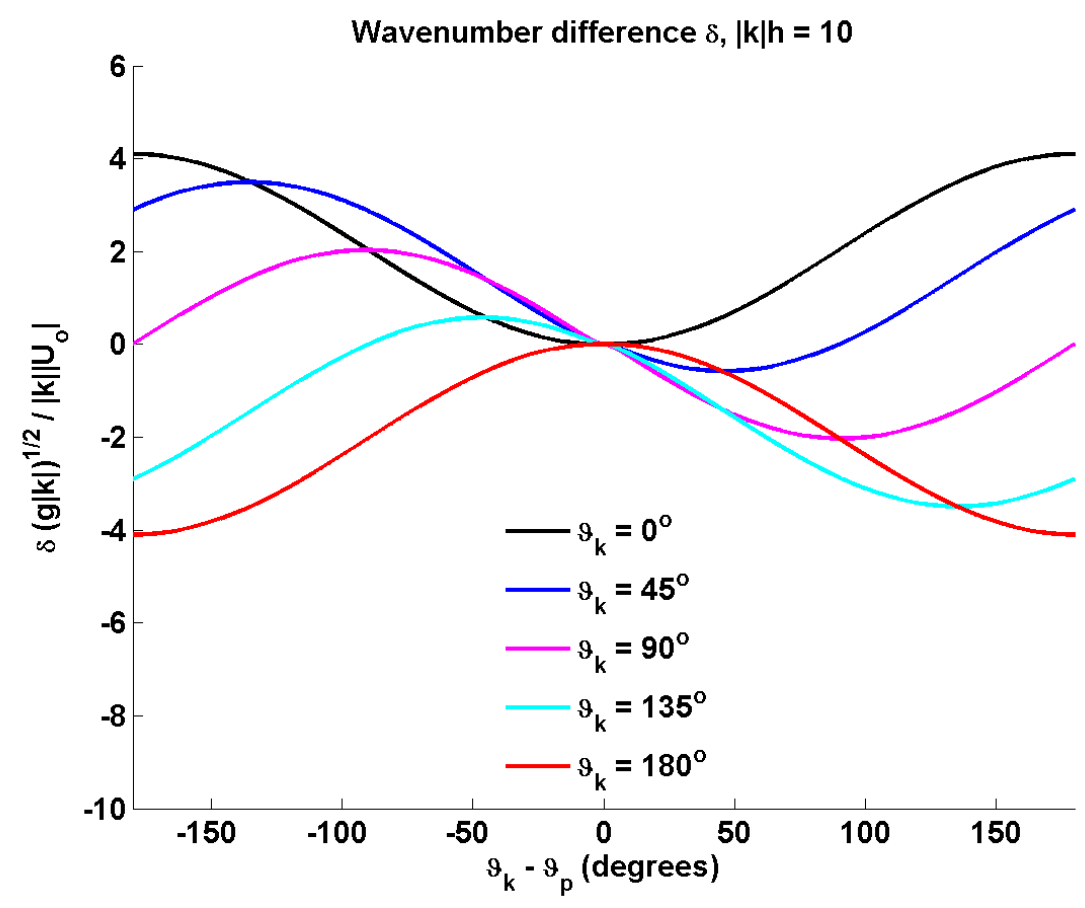

Figure 5: The solution of Equation 30 for a particular value of $h|\mathbf{k}|=10$ demonstrating how much wavenumber amplitude changes as a function of the angle between the two gravity waves.

\section{Energy Exchange in Resonant Triads}

The dynamics of resonant triads can be explored by extending the plane wave solution (Eq. 13) to include three waves which satisfy the triad condition (Eq. 1 and 2). The dynamics are assumed to evolve slowly compared to the periods of the participating waves, as in the gravity wave - interfacial wave triads of [28]. The velocity perturbation $\mathbf{u}$ and surface elevation $\eta$ are assumed to consist of three plane waves with amplitudes which slowly vary in time:

$$
\begin{array}{r}
\mathbf{u}(\mathbf{x}, z, t)=\mathfrak{R e}\left[A_{\mathbf{k}}(\epsilon t) \mathbf{v}_{\mathbf{k}}(z) \exp \left(i \mathbf{k} \cdot \mathbf{x}-i \omega_{k} t\right)+A_{\mathbf{p}}(\epsilon t) \mathbf{v}_{\mathbf{p}}(z) \exp \left(i \mathbf{p} \cdot \mathbf{x}-i \omega_{p} t\right)+A_{\mathbf{q}}(\epsilon t) \mathbf{v}_{\mathbf{q}}(z) \exp \left(i \mathbf{q} \cdot \mathbf{x}-i \omega_{q} t\right)\right] \\
\eta(\mathbf{x}, t)=\mathfrak{R e}\left[H_{\mathbf{k}}(\epsilon t) \exp \left(i \mathbf{k} \cdot \mathbf{x}-i \omega_{k} t\right)+H_{\mathbf{p}}(\epsilon t) \exp \left(i \mathbf{p} \cdot \mathbf{x}-i \omega_{p} t\right)+H_{\mathbf{q}}(\epsilon t) \exp \left(i \mathbf{q} \cdot \mathbf{x}-i \omega_{q} t\right)\right]
\end{array}
$$

Here $\epsilon$ is a small parameter, taken to be the "wave steepness", and the amplitudes $A$ and $H$ vary slowly with time. The symbol $\omega_{\mathbf{k}}$ indicates the frequency of a wave with wavenumber $\mathbf{k}$. The amplitude of the surface elevation is related to the velocity amplitude via the linearized boundary condition $H_{\mathbf{k}}(\epsilon t)=i v_{\mathbf{k}}(0) A_{\mathbf{k}}(\epsilon t) /\left(\mathbf{k} \cdot \mathbf{U}_{0}-\omega_{\mathbf{k}}\right)$. It is assumed that the three wavenumbers $(\mathbf{k}, \mathbf{q}$, and $\mathbf{p})$ satisfy the triad relationship, with $\mathbf{k}$ and $\mathbf{p}$ being gravity waves and $\mathbf{q}$ a vorticity wave, as in the previous section.

To obtain dynamical equations for the amplitudes $A$ of each constituent of the triad, the WKB-type method is applied, assuming small amplitude waves and a separation of timescales between the wave periods and the amplitude changes. Equations 31 and 32 are substituted into the free surface boundary condition. (The bulk equation of motion could in principal be used instead, but this would be inconsistent with the assumption that the amplitudes are independent of depth.) The dynamical equations for $A_{\mathbf{k}}, A_{\mathbf{q}}$ and $A_{\mathbf{p}}$ involve both resonant and non-resonant terms. The non-resonant terms describe a weak wave field about the primary resonant behavior. As is often done in the literature, all non-resonant terms are dropped from the amplitude evolution equations [28]. In some cases, this can be rigorously justified in averaged equations (over e.g. over many wave periods) and for sufficiently small $\epsilon$ so that the non-resonant terms rapidly de-phase and their contribution becomes negligible [34,35]. To prevent unphysical growth, the coefficients of these terms must balance. This leads to a set of consistency conditions in the form of a coupled system of differential equation for the amplitudes at the surface $\mathcal{A}_{\mathbf{k}}(0), \mathcal{A}_{\mathbf{p}}(0)$ and $\mathcal{A}_{\mathbf{q}}(0)$ where $\mathcal{A}(z) \equiv v_{3}(z) A$ : 


$$
\begin{aligned}
\partial_{\tau} \mathcal{A}_{\mathbf{k}} & =T_{\mathbf{k}}^{\mathbf{p q}} \mathcal{A}_{\mathbf{p}} \mathcal{A}_{\mathbf{q}} \\
\partial_{\tau} \mathcal{A}_{\mathbf{p}} & =T_{\mathbf{p}}^{\mathbf{q} \mathbf{k}} \mathcal{A}_{\mathbf{q}}^{*} \mathcal{A}_{\mathbf{k}} \\
\partial_{\tau} \mathcal{A}_{\mathbf{q}} & =T_{\mathbf{q}}^{\mathbf{k} \mathbf{p}} \mathcal{A}_{\mathbf{k}} \mathcal{A}_{\mathbf{p}}^{*}
\end{aligned}
$$

where $\tau \equiv \epsilon t$ is the slow time. Complete expressions for the interaction coefficients $T_{\mathbf{k}}^{\mathbf{p q}}$ and a further discussion of their derivation is given in Appendix C. Equations 33 governs the "leading order behavior," as for finite wave steepness there will be corrections to the dynamics of $\mathcal{A}$. The interaction coefficients $T_{\mathbf{k}}^{\mathbf{p q}}$ dictate the nature and rate of energy transfer between the waves. They can be expressed using only the current profile $\mathbf{U}$, the magnitudes $|\mathbf{k}|,|\mathbf{p}|,|\mathbf{q}|$ of the wave-vectors and their angles relative to the current $\vartheta_{k}, \vartheta_{p}, \vartheta_{q}$. Figure 6 shows how the coefficients $T_{\mathbf{k}}^{\mathbf{p q}}\left(\vartheta_{\mathbf{p}}\right), T_{\mathbf{p}}^{\mathbf{k q}}\left(\vartheta_{\mathbf{p}}\right)$ and $T_{\mathbf{q}}^{\mathbf{p k}}\left(\vartheta_{\mathbf{p}}\right)$ vary with the angle $\vartheta_{\mathbf{p}}$ in a background current with shear $\mathbf{U}^{\prime}(0)=.01 \mathrm{~s}^{-1}, \mathbf{k}$ aligned with the current $\left(\vartheta_{\mathbf{k}}=0\right)$ and $|\mathbf{k}|=1 \mathrm{~m}^{-1}$ (wavelength $\sim 6 \mathrm{~m}$ ). Eq. (33) is a Hamiltonian system provided that the frequencies are real (which is the case for almost all wave vectors at small shear) and conserves the Hamiltonian and the Manley-Rowe invariants [10]. The triad dynamics are either oscillatory (energy continually exchanged between the constituent waves) or explosive depending of the relative signs of the coefficients.

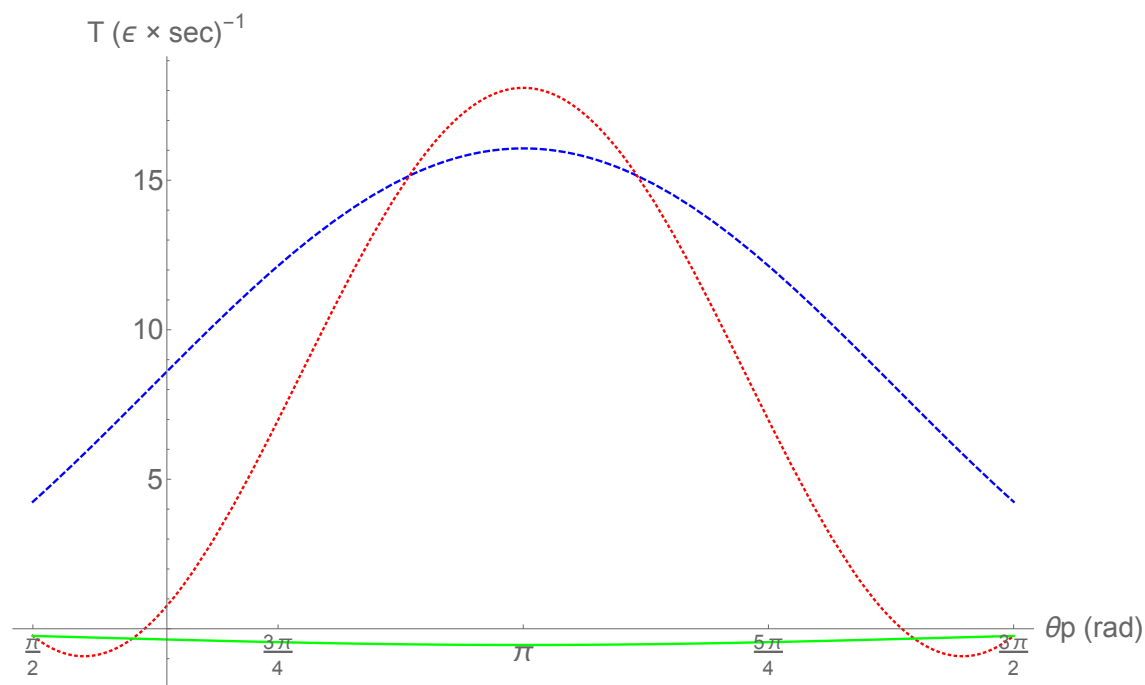

Figure 6: The three interaction coefficients for (dashed) surface wave $T_{\mathbf{k}}^{\mathbf{p q}}$, (dotted) surface wave $T_{\mathbf{p}}^{\mathbf{k q}}$ and (solid) shear mode $T_{\mathbf{q}}^{\mathbf{p k}}$ are plotted here as a function of the angle $\vartheta_{\mathbf{p}}$ of the secondary wave $\mathbf{p}$ relative to the current $\mathbf{U}$. The underlying flow has shear $\mathbf{U}^{\prime}(0)=.1 \mathrm{~s}^{-1}(\mathbf{U}(0)=1 \mathrm{~m} / \mathrm{s}$ and $h=10 \mathrm{~m})$. The primary wave number $\mathbf{k}$ is parallel to $\mathbf{U}\left(\vartheta_{\mathbf{k}}=0\right)$ with magnitude $|\mathbf{k}|=1 \mathrm{~m}^{-1}$. The coefficient for the shear wave amplitude is approximately two orders of magnitude smaller than the surface wave coefficients and is of the opposite sign (allowing for oscillating solutions of the amplitude equations).

Although semi-analytical solutions are available for the coupled triad amplitude equations in terms of Jacobi elliptic functions (see e.g. [28] for the analogous interfacial wave case), here we solve the system numerically in Mathematica using a 4th order Runge-Kutta numerical scheme. We used the software package Mathematica 10 for all of our numerical computations [17]. To limit the size of the parameter space, the direction of one gravity wave $\mathbf{k}$ is aligned with the current $\left(\vartheta_{\mathbf{k}}=0\right)$. The dynamics of the energy exchange as a function of $\varepsilon t$ is investigated as a function of the initial shear mode amplitude $\mathcal{A}_{\mathbf{q}}(-h)$ (its maximum value), the wavelength of the primary gravity wave $\lambda_{\mathbf{k}} \equiv 2 \pi /|\mathbf{k}|$, the angle between the secondary surface wave and the current $\vartheta_{\mathbf{p}}$, and the value of the surface current $\mathbf{U}_{0}$. This configuration is motivated by the oceanographic situation in which wind generates both a near-surface current and surface waves, both of which are initially aligned with the wind direction.

Physical interaction times depend on the small parameter $\varepsilon$, typically identified with wave steepness. Since steepness is not uniquely defined for a three wave system, we choose $\varepsilon$ to be combination of the three wave steepnesses 
and the evolution of the amplitudes is computed as a function of $\tau \equiv \varepsilon t$. This provides a rough measure of the energy exchange timescales for small steepness waves [28, 37, 38, 39, 40, 41].

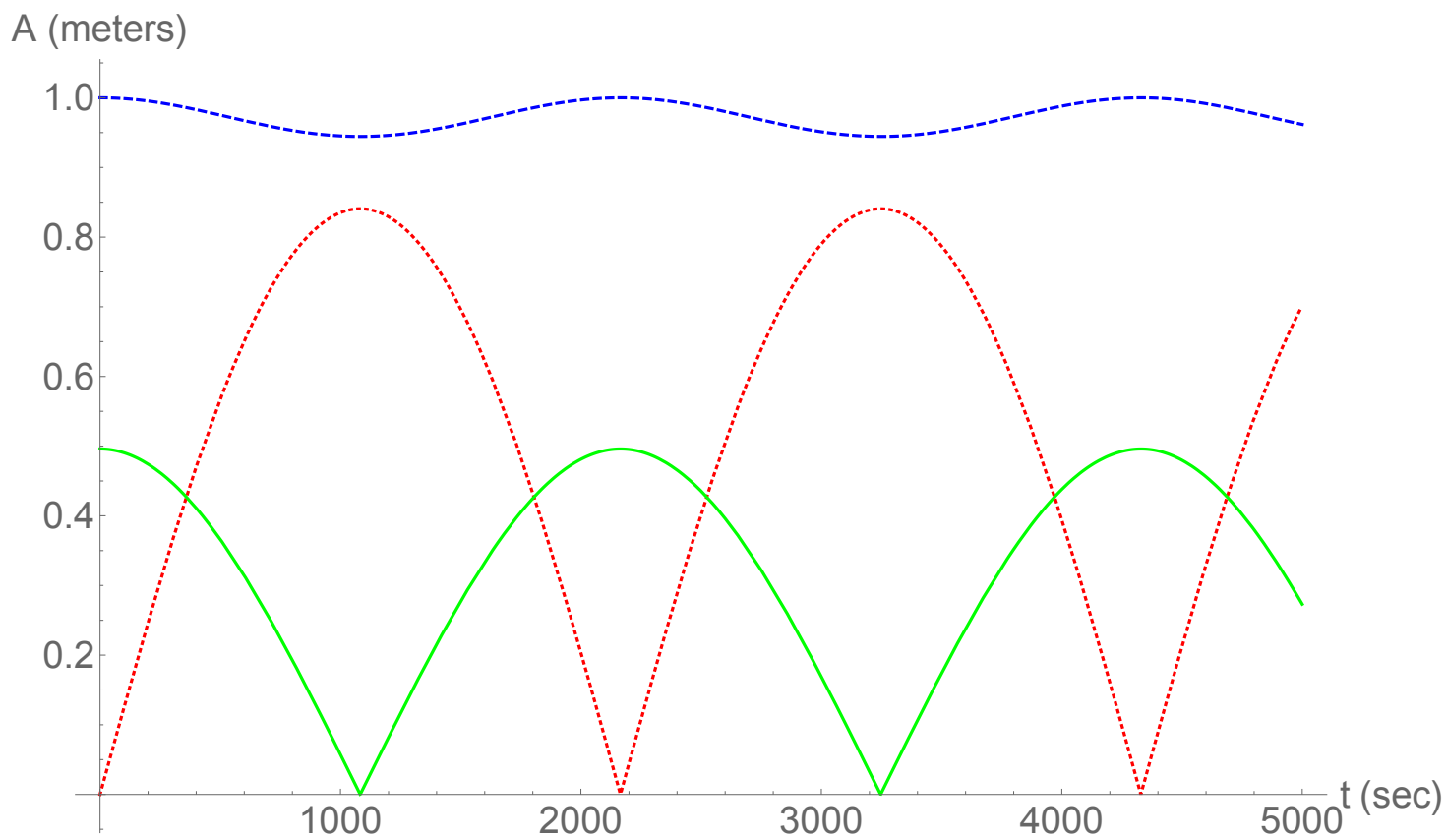

Figure 7: Example of evolutions of amplitudes $A_{\mathbf{k}}$ (primary surface wave dashed) $\mathcal{A}_{\mathbf{p}}$ (secondary surface wave dotted) and $\mathcal{A}_{\mathbf{q}}$ (shear mode solid). The waves in the example has wavelengths $|\mathbf{k}|=0.1 \mathrm{~m}^{-1}$ (wavelength $62.8 \mathrm{~m}$ ), $|\mathbf{p}|=0.09 \mathrm{~m}^{-1}$ (wavelength $62.8 \mathrm{~m}$ ) and $|\mathbf{q}|=0.05 \mathrm{~m}^{-1}$ (wavelength $122 \mathrm{~m})$ and angles to the current of $\vartheta_{\mathbf{k}}=0(\mathrm{rad}), \vartheta_{\mathbf{p}}=\pi / 6(\mathrm{rad})$ and $\vartheta_{\mathbf{q}}=1.2(\mathrm{rad})$. The initial amplitudes are $\mathcal{A}_{\mathbf{k}}(0)=1 \mathrm{~m}, \mathcal{A}_{\mathbf{p}}(0)=0$ and $\mathcal{A}_{\mathbf{q}}(-h)=.5 \mathrm{~m}$ (at kink depth of $\left.h=50 \mathrm{~m}\right)$ and the wave steepness parameter is taken to be $\varepsilon=.01$. The surface current is $\mathbf{U}(0)=0.5 \mathrm{~m} / \mathrm{s}$ with shear of $\mathbf{U}^{\prime}(0)=.01 \mathrm{~s}^{-1}$.

Figure 7 presents the wave amplitudes as a function of time for a representative triad exchange profile for a representative oceanic shear of $0.01 / \mathrm{sec}$ and $\sim 62 \mathrm{~m}$ wavelength gravity waves. The peak amplitude for each wave is shown: gravity waves at the surface and vorticity wave at the kink depth $h=50 \mathrm{~m}$ (see Fig 2 ). The primary gravity wave $\mathbf{k}$ is aligned with the current and the secondary surface wave $\mathbf{p}$ is at an angle of $\pi / 6$ to this direction and starts with zero amplitude. The vorticity wave amplitude is an order of magnitude smaller than the gravity waves at the surface and $\sim 0.5 \mathrm{~m}$ at the kink depth. Figure 7 illustrates how two gravity waves at different angles to each other may exchanging energy, substantially changing their amplitude in minutes to hours. This suggests that subsurface currents may facilitate the directional spreading of wind generated gravity waves. In oceanographic applications, the primary gravity wave would also be receiving energy input from the wind and also interacting with other waves in the spectrum, so the dynamics would not be simply oscillatory, but the timescales for energy transfer would be comparable to those seen in this example.

In Figure 8, we study dependency of energy transfer rates on varying underlying parameters. In each case, only the secondary gravity wave amplitude $\mathcal{A}_{\mathbf{p}}(0)$ is shown. Figure 8 a illustrates how changing the background shear affects the energy exchange timescale: as the background shear is increased, energy exchange becomes more rapid and the amplitudes increase. This observation is more or less intuitive; a larger shear, induced by a stronger wind, facilitates faster exchange. The effect of wavelength on energy exchange is illustrated in Figure 8b: as wavelength decreases, energy exchange becomes more rapid. Hence shear-mediated energy exchange appears to be consistent with the oceanic observation that directional spreading of wind waves occurs primarily for shorter wavelengths $[6,7$, 8]. Although the secondary wave amplitude decreases with wavelength, the wave steepness $|\mathbf{p}| \mathcal{A}_{\mathbf{p}}(0)$ is approximately constant. Figure 8c illustrates the dynamics for different values of the secondary gravity wave angle $\vartheta_{\mathrm{p}}$. As $\vartheta_{\mathrm{p}}$ increases, net energy exchange slows down and the peak amplitude of $\mathcal{A}_{\mathbf{p}}(0)$ decreases. This indicates that energy exchange is most efficient for relatively small angles, which is again consistent with observed wave spreading. Finally, Figure $8 \mathrm{~d}$ presents the dependence of $\mathcal{A}_{\mathbf{p}}(0)$ on the vorticity wave initial amplitude $\mathcal{A}_{\mathbf{q}}(-h)$ : decreasing the initial 
vorticity mode amplitude reduces total energy transfer.

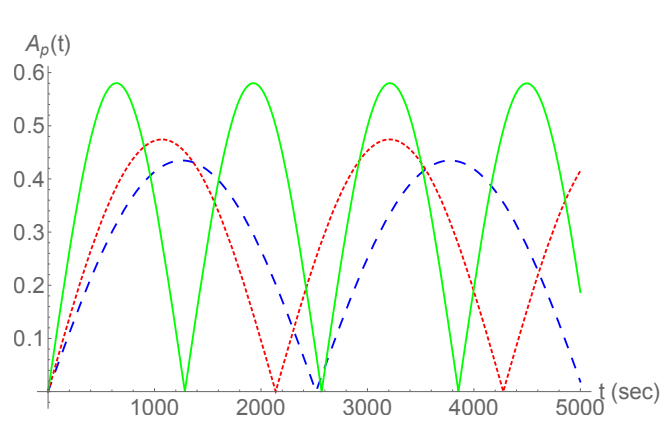

(a) Dependence on surface shear strength. The dashed curve corresponds to $\mathbf{U}^{\prime}(0)=0.005 / \mathrm{s}$, the dotted to $\mathbf{U}^{\prime}(0)=0.013 / \mathrm{s}$ and the solid to $\mathbf{U}^{\prime}(0)=0.1 / \mathrm{s}$.

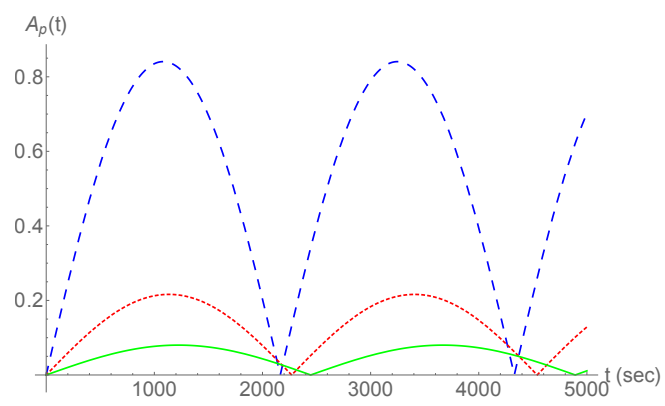

(c) Dependence on angle of secondary gravity wave to the current. (d) The dashed curve corresponds to $\vartheta_{\mathbf{p}}=\pi / 6$, the dotted to $\vartheta_{\mathbf{p}}=\pi / 4$ and the solid to $\vartheta_{\mathrm{p}}=\pi / 3$.

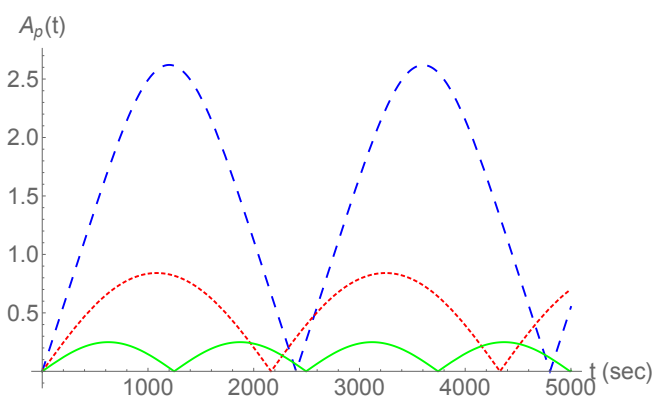

(b) Dependence on primary gravity wave wavelength. The dashed curve corresponds to $|\mathbf{k}|$ of 0.07 (wavelength $=89.7 \mathrm{~m}$ ), the dotted to $|\mathbf{k}|=0.1$ (wavelength $=62.8 \mathrm{~m}$ ) and the solid to $|\mathbf{k}|=0.2$ (wavelength $=31.4 \mathrm{~m}$ ).

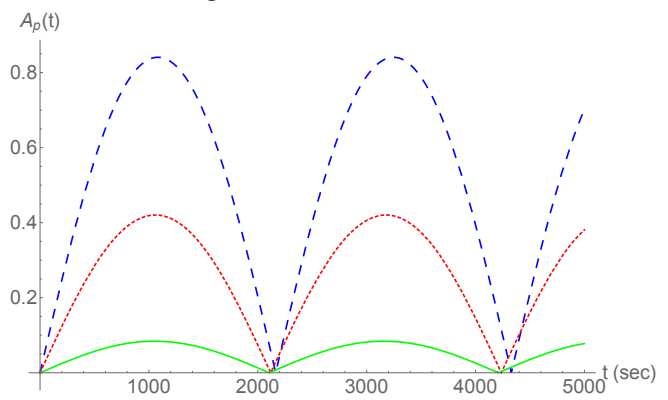

(d) Dependence on initial vorticity mode amplitude. The dashed curve corresponds to $\mathcal{A}_{\mathbf{q}}(-h)=0.5 \mathrm{~m}$, the dotted to $\mathcal{A}_{\mathbf{q}}(-h)=$ $0.25 \mathrm{~m}$ and the solid to $\mathcal{A}_{\mathbf{q}}(-h)=0.05 \mathrm{~m}$.

Figure 8: Effects of varying parameters on the rate of energy transfer within the wave triad. In each subplot, all parameters except the one being varied are kept the same as in Figure 7 . For brevity, only the secondary gravity wave amplitude $\mathcal{A}_{\mathbf{p}}(0)$ as a function of changing parameters is shown.

\section{Discussion}

The focus of this work was to study the effect of oceanic underwater currents on gravity waves. Weakly nonlinear theory was used to show that, for an idealized current profile in three dimensions, resonant triads involving two gravity waves and a sub-surface vorticity wave are possible. These triads connect a pair of gravity waves with comparable wavelengths but different directions of propagation. Weakly nonlinear theory was then employed to investigate the rate of energy exchange associated with these triads. The specific scenario considered energy transfer from one gravity wave to another gravity wave with initially small amplitude. This configuration was motivated by the observed spreading of wind-waves away from the direction of initial energy input (aligned with the wind). The energy transfer trends indicated by this analysis are:

- For gravity waves traveling at angles to each other, a shear mediated triad exchange forces one gravity wave to gain energy at the expense of the other in a quasi-periodic way.

- Generally, energy exchange rates are increased if the shear increases, the wavelength of the gravity waves decreases, or the angle the secondary wave to the primary is decreased. The wavelength dependence could possibly explain why longer waves in a wind-sea spectrum are primarily windward while the shorter waves are more omni-directional. 
- For physically reasonable surface currents, shears and wavelengths, triads exchange energy occurs on time scales of tens of minutes to many hours (depending on the particular chosen parameters). Given gravity wave speeds of a few meters per second, this indicates that the shear interaction region would have to be at least a few kilometers in horizontal extent for significant energy exchange to occur.

It is important to discuss the limitations of these results and the model used to obtain them. The piecewise linear profile with one kink is an idealized representation of realistic ocean currents, and results will differ from the true ocean environment. It is intended only to qualitatively investigate the implications of resonant triads involving shear, and probably is not quantitatively accurate. The profile also permits only a single vorticity wave mode, while realistic currents may exhibit a spectrum of modes, possibly allowing additional triads. Also, horizontal variations in the shear were neglected, and the shear was assumed to have infinite horizontal extent. Rotation is also not considered. Therefore, the results presented here should be viewed as a preliminary investigation and exposition on shear mediated non-linear mechanisms of energy transfer among ocean gravity waves. The predictions made here should be thought of as rough estimates to the magnitudes and trends of the shear-mediated interactions, but should not be assumed to be quantitatively precise. Future work could explore the vorticity wave dispersion relation for more realistic current profiles, including rotation, using numerical solutions. It would be valuable to establish what characteristic(s) of the current profile are necessary for vorticity waves to participate in triads with gravity waves, and to assess the prevalence of these conditions in the oceans.

This study was performed assuming a single resonant triad interaction between plane waves of infinite horizontal extent. This won't be the case in the ocean, where a continuous spectrum of gravity waves may permit many triad resonances. Numerical simulations will be needed to more fully explore the implications of the triad interactions considered here for ocean waves. One possible approach will be to develop a weakly-nonlinear pseudo-spectral numerical simulation analogous to the approach of Kent and Choi [43] for potential flow. The subsurface solutions for kinked profiles presented here would be used to formulate dynamical equations using only surface variables to 2nd order in the weakly nonlinear expansion. This approach would include all possible weakly nonlinear interactions between surface and vorticity waves. Results could be used to assess the importance of wave triads relative to other interactions. Alternately, a wave kinetic theory could be used to investigate triad interactions in a fully developed spectrum. Necessary steps include deriving the full kinetic equation for shear-gravity wave turbulence and numerically computing the spectrum. Rotation would have to be included, as well as surface tension for shorter wavelengths (which have faster interactions).

\section{Acknowledgements}

T.D. would like to thank Nikos Bakas, Oliver Bühler, and Navid Constantinou for useful discussions. The authors would also like to thank the anonymous referees for their many helpful comments and suggestions. This work was supported by the Defense Advanced Research Projects Agency. The views expressed are those of the authors and do not reflect the official policy or position of the Department of Defense or the U.S. Government.

\section{Appendix A. Derivation of Surface Boundary Conditions}

\section{Appendix A.1. Free Surface Condition}

The equation the surface elevation satisfies, (8), is highly implicit because each term appearing must be evaluated at the yet undetermined surface. To circumvent this difficulty, we assume the deformation is small and Taylor expand about the equilibrium position of the surface $z=0$. To this purpose, define the operator:

$$
\left.\mathcal{L}_{\eta}[f] \equiv\left(1+\eta \partial_{z} f+\frac{1}{2 !} \eta^{2} \partial_{z}^{2} f+\frac{1}{3 !} \eta^{3} \partial_{z}^{3} f+\cdots\right)\right|_{z=0}
$$

Our objective is to work to second order in a wave steepness expansion of the surface elevation and velocity perturbation. We therefore Taylor expand necessary terms in (8) but drop all interactions of three or more fields. Explicitly we have

$$
\mathcal{L}_{\eta}\left[D_{t} \eta\right]+\mathcal{L}_{\eta}\left[\mathbf{u} \cdot \nabla_{\mathrm{H}} \eta-u_{3}\right]=0
$$

which, at second order yields Eq. (9). 


\section{Appendix A.2. Velocity Boundary Condition}

To find the condition $u_{3}$ must satisfy at the free surface we will combine the kinematic boundary condition and the dynamic boundary condition. Taking the horizontal Laplacian of the dynamic condition we have:

$$
g \Delta_{\mathrm{H}} \eta=\Delta_{\mathrm{H}}(p / \rho)+2 \nabla_{\mathrm{H}} \eta \cdot \nabla_{\mathrm{H}} \partial_{z}(p / \rho)+\left|\nabla_{\mathrm{H}} \eta\right|^{2} \partial_{z}^{2}(p / \rho)+\partial_{z}(p / \rho) \Delta_{\mathrm{H}} \eta,
$$

where each term evaluated on the free surface $z=\eta(x, y, t)$. Using the Euler equations to substitute out the pressure terms, we find that:

$$
\begin{aligned}
g \Delta_{\mathrm{H}} \eta= & D_{t} \partial_{z} u_{3}-\mathbf{U}^{\prime} \cdot \nabla_{\mathrm{H}} u_{3}-2 \nabla_{\mathrm{H}} \eta \cdot\left(D_{t} \nabla_{\mathrm{H}} u_{3}+\nabla_{\mathrm{H}}\left[\mathbf{u} \cdot \nabla u_{3}\right]\right) \\
& -\left|\nabla_{\mathrm{H}} \eta\right|^{2}\left(D_{t} \partial_{z} u_{3}+\mathbf{U}^{\prime} \cdot \nabla_{\mathrm{H}} u_{3}+\partial_{z}\left[\mathbf{u} \cdot \nabla u_{3}\right]\right) \\
& -\Delta_{\mathrm{H}} \eta\left(D_{t} u_{3}+\mathbf{u} \cdot \nabla u_{3}\right)-\nabla_{\mathrm{H}} \cdot[\mathbf{u} \cdot \nabla \mathbf{u}]
\end{aligned}
$$

on the free surface $z=\eta(x, y, t)$. Keeping only linear and quadratic terms we have:

$$
\begin{aligned}
g \Delta_{\mathrm{H}} \eta & =D_{t} \partial_{z} u_{3}-\mathbf{U}^{\prime} \cdot \nabla_{\mathrm{H}} u_{3}-\mathcal{I}_{1}(\mathbf{u}, \eta) \\
\mathcal{I}_{1}(\mathbf{u}, \eta) & \equiv \nabla_{\mathrm{H}} \cdot[\mathbf{u} \cdot \nabla \mathbf{u}]+2 \nabla_{\mathrm{H}} \eta \cdot D_{t} \nabla_{\mathrm{H}} u_{3}+\Delta_{\mathrm{H}} \eta D_{t} u_{3}
\end{aligned}
$$

In anticipation of combining the above with the free surface condition, we take the material derivative:

$$
\begin{aligned}
g D_{t} \Delta_{\mathrm{H}} \eta= & D_{t}^{2} \partial_{z} u_{3}-\mathbf{U}^{\prime} \cdot D_{t} \nabla_{\mathrm{H}} u_{3}-\mathcal{I}_{2}(\mathbf{u}, \eta) \\
\mathcal{I}_{2}(\mathbf{u}, \eta) \equiv & \nabla_{\mathrm{H}} \cdot D_{t}[\mathbf{u} \cdot \nabla \mathbf{u}]+D_{t} \eta D_{t} \Delta_{\mathrm{H}} u_{3}+2 D_{t} \nabla_{\mathrm{H}} \eta \cdot D_{t} \nabla_{\mathrm{H}} u_{3} \\
& +2 \nabla_{\mathrm{H}} \eta \cdot D_{t}^{2} \nabla_{\mathrm{H}} u_{3}+D_{t} \Delta_{\mathrm{H}} \eta D_{t} u_{3}+\Delta_{\mathrm{H}} \eta D_{t}^{2} u_{3}
\end{aligned}
$$

It should be noted that the material differentiation in $D_{t}$, by the chain rule brings factors of $D_{t} \eta$ from $\left.\mathbf{U}\right|_{z=\eta}$ and $\left.\mathbf{u}\right|_{z=\eta}$ terms. The terms which appear can be simplified $D_{t} \eta\left[D_{t} \partial_{z}^{2} u_{3}-\mathbf{U}^{\prime \prime} \cdot \nabla_{\mathrm{H}} u_{3}\right]=-D_{t} \eta D_{t} \Delta_{\mathrm{H}} u_{3}$ by Equation (5) if we keep only quadratic terms in the fields. Continuing on the path to find the boundary condition for $u_{3}$, we take the horizontal Laplacian of the kinematic boundary condition. Keeping only terms quadratic in the fields we have:

$$
\begin{aligned}
D_{t} \Delta_{\mathrm{H}} \eta & =\Delta_{\mathrm{H}}\left[u_{3}-\mathbf{u} \cdot \nabla_{\mathrm{H}} \eta\right]=\Delta_{\mathrm{H}} u_{3}+\mathcal{I}_{3}(\mathbf{u}, \eta) \\
\mathcal{I}_{3}(\mathbf{u}, \eta) & \equiv 2 \nabla_{\mathrm{H}} \eta \cdot \nabla_{\mathrm{H}} \partial_{z} u_{3}+\partial_{z} u_{3} \Delta_{\mathrm{H}} \eta-2 \partial_{i} \delta u_{j} \partial_{i} \partial_{j} \eta-\mathbf{u} \cdot \nabla_{\mathrm{H}}\left(\Delta_{\mathrm{H}} \eta\right)-\left(\Delta_{\mathrm{H}} \mathbf{u}\right) \cdot \nabla_{\mathrm{H}} \eta
\end{aligned}
$$

Equating like terms in (A.3) and (A.5) we find the condition to hold on the moving surface is:

$$
D_{t}^{2} \partial_{z} u_{3}-\mathbf{U}^{\prime} \cdot D_{t} \nabla_{\mathrm{H}} u_{3}-g \Delta_{\mathrm{H}} u_{3}=g \mathcal{I}_{3}(\mathbf{u}, \eta)-\mathcal{I}_{2}(\mathbf{u}, \eta) \quad \text { on } \quad z=\eta(x, y, t) .
$$

As before, we now expand around the equilibrium position of the surface at $z=0$. Applying the operator $\mathcal{L}_{\eta}$ given by Equation (A.1) to (A.6) and keeping only up to quadratic order in the fields we have:

$$
D_{t}^{2} \partial_{z} u_{3}-\mathbf{U}^{\prime} \cdot \nabla_{\mathrm{H}} D_{t} u_{3}-\left.g \Delta_{\mathrm{H}} u_{3}\right|_{z=0}=\left[g \eta \Delta_{\mathrm{H}} \partial_{z} u_{3}+g \mathcal{I}_{3}(\mathbf{u}, \eta)-\mathcal{I}_{2}(\mathbf{u}, \eta)-\eta \partial_{z}\left(D_{t}^{2} \partial_{z} u_{3}-\mathbf{U}^{\prime} \cdot \nabla_{\mathrm{H}} D_{t} u_{3}\right)\right]_{z=0}
$$

We go on to simply the above expression. Using (5), one can show, to linear order, that

$$
\partial_{z}\left(D_{t} \partial_{z} u_{3}-\mathbf{U}^{\prime}(z) \cdot \nabla u_{3}\right)+D_{t} \Delta_{\mathrm{H}} u_{3}=0 .
$$

Taking the material derivative of the above we find the relationship 


$$
\partial_{z}\left(D_{t}^{2} \partial_{z} u_{3}-\mathbf{U}^{\prime}(z) \cdot \nabla D_{t} u_{3}\right)=-D_{t}^{2} \Delta_{\mathrm{H}} u_{3}-\mathbf{U}^{\prime}(z) \cdot \nabla_{\mathrm{H}}\left(D_{t} \partial_{z} u_{3}-\mathbf{U}^{\prime}(z) \cdot \nabla u_{3}\right)
$$

and so, upon substitution we arrive at the boundary condition for the vertical velocity perturbation to second order in the fields:

$$
D_{t}^{2} \partial_{z} u_{3}-\mathbf{U}^{\prime} \cdot \nabla_{\mathrm{H}} D_{t} u_{3}-g \Delta_{\mathrm{H}} u_{3}=\eta\left(\mathbf{U}^{\prime} \cdot \nabla_{\mathrm{H}}\left(D_{t} \partial_{z} u_{3}-\mathbf{U}^{\prime} \cdot \nabla u_{3}\right)+D_{t}^{2} \Delta_{\mathrm{H}} u_{3}+g \Delta_{\mathrm{H}} \partial_{z} u_{3}\right)+\mathcal{I}_{2}(\mathbf{u}, \eta)+g \mathcal{I}_{3}(\mathbf{u}, \eta),
$$

all evaluated at $z=0$.

\section{Appendix B. Matching conditions}

In this section we will derive a set of matching conditions for the solution of the linearization of (5) on the fixed domain $\mathcal{D}:=\mathbb{R}^{2} \times \mathbb{R}^{-} \times[0, T)$. when the underlying current profile $\mathbf{U}(z)$ is piecewise linear. Suppose that on $\mathbf{U}^{\prime}$ is discontinuous on the set of $z$ values $\mathcal{S}:=\left\{z_{i}\right\}_{i=1}^{n}$. Define the sets $C_{i}:=\mathbb{R}^{2} \times\left[z_{i-1}, z_{i}\right) \times[0, T)$ for $n \geq i \geq 1$, $C_{0}=\mathbb{R}^{2} \times\left(-\infty, z_{1}\right) \times[0, T)$ and $C_{n+1}=\mathbb{R}^{2} \times\left(z_{n}, 0\right] \times[0, T)$. It follows that $\mathcal{D} \backslash \mathcal{S}=\bigcup_{i=0}^{n+1} \mathcal{C}_{i}$. We say that $u_{3}$ is a weak solution if, for all smooth compactly supported (on the domain $\mathcal{D}$ ) test functions $\varphi \equiv \varphi(\mathbf{x}, t)$, the following equality is satisfied:

$$
\begin{gathered}
\int_{\mathcal{D}}\left(D_{t}\left(\partial_{z}^{2}+\Delta_{\mathrm{H}}\right) u_{3}^{(1)}-\mathbf{U}^{\prime \prime}(z) \cdot \nabla_{\mathrm{H}} u_{3}^{(1)}\right) \varphi=0, \\
\int_{\mathcal{D}}\left(\nabla_{\mathrm{H}} \cdot u_{i}^{(1)}+\partial_{z} u_{3}^{(1)}\right) \varphi=0 .
\end{gathered}
$$

The first equation is the linearized momentum balance whereas the second is the incompressibility constraint. There is an equation for $u_{i}^{(1)}$ which is in principle necessary to state the complete problem but it is not needed in our analysis so it is omitted. Since we may change the domain of integration by a measure zero set without effecting the integral, we can integrate over all the $C_{i}$ 's instead of $\mathcal{D}$ in equation (B.1). Using Equation (A.7), we write (B.1) as

$$
\left.\sum_{i=0}^{n} \int_{\mathcal{D} \backslash \mathbb{R}^{-}} \mathrm{d} t \mathrm{~d} \mathbf{x}_{\mathrm{H}}\left(D_{t} \partial_{z} u_{3}^{(1)}-\mathbf{U}^{\prime}(z) \cdot \nabla u_{3}^{(1)}\right) \varphi\right|_{z_{i}} ^{z^{i+1}}-\int_{\mathcal{D}}\left[\left(D_{t} \partial_{z} u_{3}^{(1)}-\mathbf{U}^{\prime}(z) \cdot \nabla u_{3}^{(1)}\right) \partial_{z} \varphi-\left(D_{t} \Delta_{\mathrm{H}} u_{3}^{(1)}\right) \varphi\right]=0
$$

where we have performed an integration by parts in the $z$ coordinate and named $z_{0}=-\infty$ and $z_{n+1}=0$. The boundary terms appear at all the $z$-endpoints of the disconnected regions of $\cup \mathcal{C}_{i}$. The terms at infinity vanish since $\varphi$ is of compact support. The second integral is automatically zero since $u_{3}$ is a weak solution satisfying equation (B.1). We are left with the condition that the boundary terms vanish:

$$
\left.\sum_{i=0}^{n} \int_{\mathcal{D} \backslash \mathbb{R}^{-}} \mathrm{d} t \mathrm{~d} \mathbf{x}_{\mathrm{H}}\left(D_{t} \partial_{z} u_{3}^{(1)}-\mathbf{U}^{\prime}(z) \cdot \nabla u_{3}^{(1)}\right) \varphi\right|_{z_{i}} ^{z^{i+1}}=0
$$

For $u_{3}$ to be a weak solution, we require that this be satisfied for all test functions $\varphi$. In particular, choosing a set of test functions $\varphi_{i}$ each with support containing the point $z_{i}$ for $i=1, \ldots, n$ but no other points in $\mathcal{S}$, the above condition simplifies to

$$
\left.\lim _{\epsilon \rightarrow 0} \int_{\mathcal{D} \backslash \mathbb{R}^{-}} \mathrm{d} t \mathrm{~d} \mathbf{x}_{\mathrm{H}}\left(D_{t} \partial_{z} u_{3}^{(1)}-\mathbf{U}^{\prime}(z) \cdot \nabla u_{3}^{(1)}\right) \varphi_{i}\right|_{z_{i}-\epsilon} ^{z_{i}+\epsilon}=0
$$

Again, since this holds for all $\varphi_{i}$, the we conclude that $\left.\lim _{\epsilon \rightarrow 0}\left(D_{t} \partial_{z} u_{3}^{(1)}-\mathbf{U}^{\prime}(z) \cdot \nabla u_{3}^{(1)}\right)\right|_{z_{i}-\epsilon} ^{z_{i}+\epsilon}=0$ must hold for each point $z_{i} \in \mathcal{S}$. Repeating this procedure but now for the continuity equation (B.2), one finds $\left.\lim _{\epsilon \rightarrow 0}\left(u_{3}^{(1)}\right)\right|_{z_{i}-\epsilon} ^{z_{i}+\epsilon}=0$ must also be satisfied for each $z_{i} \in \mathcal{S}$. For the problem of one kink at depth $z=-h$, the matching conditions read: 


$$
\begin{array}{r}
{\left[\left[u_{3}^{(1)}\right]\right]_{z=-h}=0,} \\
{\left[\left[D_{t} \partial_{z} u_{3}^{(1)}-\mathbf{U}^{\prime}(z) \cdot \nabla u_{3}^{(1)}\right]\right]_{z=-h}=0,}
\end{array}
$$

where $[[f]]_{z=x}=f^{+}(x)-f^{-}(x)$ is the jump in the function $f(z)$ across the point $z=x$. The same analysis can be conducted for the second order in $\mu$ equations and the same matching conditions, appropriately modified, apply.

\section{Appendix C. Triad Resonance Equations}

A straightforward but lengthy calculation is required to derive Equations (33). We have in mind the situation where we have a wave train solution that has slow, as well as fast, space and time dependence:

$$
\mathbf{u}(\mathbf{x}, t)=\epsilon \mathbf{u}^{(1)}(\epsilon t, \epsilon \mathbf{x}, t, \mathbf{x})+\epsilon^{2} \mathbf{u}^{(2)}(\epsilon t, \epsilon \mathbf{x}, t, \mathbf{x})+\cdots
$$

for $\epsilon \ll 1$. The boundary condition Eq. (10) for wave train solutions with slowly varying amplitudes (31) becomes:

$$
\left(2 D_{t} \widetilde{D}_{\tau} \partial_{z}-\mathbf{U}^{\prime} \cdot \widetilde{\nabla}_{\mathrm{H}} D_{t}-\mathbf{U}^{\prime} \cdot \nabla_{\mathrm{H}} \widetilde{D}_{\tau}-2 g \widetilde{\nabla}_{H} \cdot \nabla_{H}\right) u_{3}^{(1)}=-\left(D_{t}^{2} \partial_{z}-\mathbf{U}^{\prime} \cdot \nabla_{\mathrm{H}} D_{t}-g \Delta_{\mathrm{H}}\right) u_{3}^{(2)}+\mathcal{N}\left(\mathbf{u}^{(1)}, \eta^{(1)}\right)
$$

where a tilde over an operator indicates that it acts on the "slow" $\epsilon t$ or $\epsilon \mathbf{x}$ variables. Assume that the perturbation $\mathbf{u}$ is composed of three monochromatic waves $\mathbf{k}, \mathbf{p}, \mathbf{q}$ satisfying the resonant triad conditions $\mathbf{k}=\mathbf{p}+\mathbf{q}, \omega_{\mathbf{k}}=\omega_{\mathbf{p}}+\omega_{\mathbf{q}}$. We arrive at three coupled partial differential equations by substituting this sum of the monochromatic waves (31) into the above equation, keeping only resonant interaction. A simplification occurs if we assume that there is no dependence on the slow spatial variable $\epsilon \mathbf{x}$. Further, terms proportional to the second order velocity (since $\mathbf{u}^{(2)} \sim e^{i 2 \mathbf{k} \cdot \mathbf{x}-2 i \omega t}$ ) are non-resonant and therefore dropped. We are left with the following set of differential equations:

$$
\begin{aligned}
& \left(2 D_{t} \widetilde{D}_{\tau} \partial_{z}-\mathbf{U}^{\prime} \cdot \nabla_{\mathrm{H}} \widetilde{D}_{\tau}\right) u_{3}^{\mathbf{k}}=\mathcal{N}\left(\mathbf{u}^{\mathbf{q}}, \eta^{\mathbf{p}}\right)+\mathcal{N}\left(\mathbf{u}^{\mathbf{p}}, \eta^{\mathbf{q}}\right) \\
& \left(2 D_{t} \widetilde{D}_{\tau} \partial_{z}-\mathbf{U}^{\prime} \cdot \nabla_{\mathrm{H}} \widetilde{D}_{\tau}\right) u_{3}^{\mathbf{p}}=\mathcal{N}\left(\mathbf{u}^{\mathbf{q}}, \eta^{\mathbf{k}}\right)+\mathcal{N}\left(\mathbf{u}^{\mathbf{k}}, \eta^{\mathbf{q}}\right) \\
& \left(2 D_{t} \widetilde{D}_{\tau} \partial_{z}-\mathbf{U}^{\prime} \cdot \nabla_{\mathrm{H}} \widetilde{D}_{\tau}\right) u_{3}^{\mathbf{q}}=\mathcal{N}\left(\mathbf{u}^{\mathbf{k}}, \eta^{\mathbf{p}}\right)+\mathcal{N}\left(\mathbf{u}^{\mathbf{p}}, \eta^{\mathbf{k}}\right)
\end{aligned}
$$

where all the perturbations are first order, $\mathbf{u}^{\mathbf{k}}=\Re \mathfrak{R} A_{\mathbf{k}}(\epsilon t) e^{i \mathbf{k} \cdot \mathbf{x}-i \omega t}$ indicating a monochromatic wave with wavenumber k. The nonlinear terms (written out explicitly in Eq. (11)) will produce many waves which will not be resonant. Equations (33) are obtained by the process of substitution, keeping only terms proportional to $e^{i \mathbf{k} \cdot \mathbf{x}-i \omega t}$ in the equation for $\mathcal{A}_{\mathbf{k}}, e^{i \mathbf{p} \cdot \mathbf{x}-\omega t}$ for $\mathcal{A}_{\mathbf{p}}$, etc. In, for example, the equation for $A_{\mathbf{k}}$, the terms which are resonant will give a contribution to the dynamics of the form $T_{\mathbf{p q}}^{\mathbf{k}} A_{\mathbf{q}} A_{\mathbf{p}}$ where $T_{\mathbf{p q}}^{\mathbf{k}}$, found by direct computation, are: 


$$
\begin{aligned}
& T_{\mathbf{k}}^{\mathbf{p q}} \equiv \frac{|\mathbf{k}|}{\Omega_{0}^{\mathbf{q}} \Omega_{0}^{\mathbf{p}}} \frac{\Omega_{0}^{\mathbf{k}}}{\left(1-\beta_{0}^{\mathbf{k}} \Omega_{0}^{\mathbf{k}}\right)}\left[\frac{\left(\omega_{0}^{\mathbf{p}}\right)^{2}}{\omega_{0}^{\mathbf{k}} \omega_{0}^{\mathbf{q}}} \frac{|\mathbf{p}|^{2}}{|\mathbf{k}|^{2}} \beta_{0}^{\mathbf{p}}+\frac{\left(\omega_{0}^{\mathbf{q}}\right)^{2}}{\omega_{0}^{\mathbf{k}} \omega_{0}^{\mathbf{p}}} \frac{|\mathbf{q}|^{2}}{|\mathbf{k}|^{2}} \beta_{0}^{\mathbf{q}}\right] \\
& -\frac{|\mathbf{k}|}{2} \frac{\Omega_{0}^{\mathbf{k}}}{\omega_{0}^{\mathbf{k}}\left(1-\beta_{0}^{\mathbf{k}} \Omega_{0}^{\mathbf{k}}\right)}\left[-\omega_{0}^{\mathbf{p}} \Omega_{0}^{\mathbf{p}}\left(\frac{\omega_{0}^{\mathbf{p}} \Omega_{0}^{\mathbf{p}}}{\omega_{0}^{\mathbf{q}} \Omega_{0}^{\mathbf{q}}}+1\right)+\frac{\omega_{0}^{\mathbf{k}} \Omega_{0}^{\mathbf{k}}}{|\mathbf{k}|^{2} v_{3}^{\mathbf{q}} v_{3}^{\mathbf{p}}}\left(\mathbf{q} \cdot v_{H}^{\mathbf{p}} v_{3}^{\mathbf{q}^{\prime}}-\mathbf{q} \cdot v_{H}^{\mathbf{p}} \mathbf{p} \cdot v_{H}^{\mathbf{q}}+|\mathbf{q}|^{2} v_{3}^{\mathbf{p}} v_{3}^{\mathbf{q}}-\mathbf{p} \cdot v_{H}^{\mathbf{q}{ }^{\prime}} v_{3}^{\mathbf{p}}\right)\right] \\
& -\frac{|\mathbf{k}|}{2} \frac{\Omega_{0}^{\mathbf{k}}}{\omega_{0}^{\mathbf{k}}\left(1-\beta_{0}^{\mathbf{k}} \Omega_{0}^{\mathbf{k}}\right)}\left[-\omega_{0}^{\mathbf{q}} \Omega_{0}^{\mathbf{q}}\left(\frac{\omega_{0}^{\mathbf{q}} \Omega_{0}^{\mathbf{q}}}{\omega_{0}^{\mathbf{p}} \Omega_{0}^{\mathbf{p}}}+1\right)+\frac{\omega_{0}^{\mathbf{k}} \Omega_{0}^{\mathbf{k}}}{|\mathbf{k}|^{2} v_{3}^{\mathbf{q}} v_{3}^{\mathbf{p}}}\left(\mathbf{p} \cdot v_{H}^{\mathbf{q}} v_{3}^{\mathbf{p}{ }^{\prime}}-\mathbf{p} \cdot v_{H}^{\mathbf{q}} \mathbf{q} \cdot v_{H}^{\mathbf{p}}+|\mathbf{p}|^{2} v_{3}^{\mathbf{q}} v_{3}^{\mathbf{p}}-\mathbf{q} \cdot v_{H}^{\mathbf{p}}{ }^{\prime} v_{3}^{\mathbf{q}}\right)\right] \\
& -\frac{|\mathbf{k}|}{2} \frac{\Omega_{0}^{\mathbf{k}}}{\omega_{0}^{\mathbf{k}}\left(1-\beta_{0}^{\mathbf{k}} \Omega_{0}^{\mathbf{k}}\right)}\left[\frac{\left(\omega_{0}^{\mathbf{p}}\right)^{2}}{\omega_{0}^{\mathbf{q}} \Omega_{0}^{\mathbf{q}}} \frac{1}{v_{3}^{\mathbf{p}}(0)}\left(\frac{v_{3}^{\mathbf{p}^{\prime}}}{|\mathbf{p}|}-\frac{\mathbf{q} \cdot v_{H}^{\mathbf{p}}}{|\mathbf{p}|}\right)+\frac{\left(\omega_{0}^{\mathbf{q}}\right)^{2}}{\omega_{0}^{\mathbf{p}} \Omega_{0}^{\mathbf{p}}} \frac{1}{v_{3}^{\mathbf{q}}(0)}\left(\frac{v_{3}^{\mathbf{q}^{\prime}}}{|\mathbf{q}|}-\frac{\mathbf{p} \cdot v_{H}^{\mathbf{q}}}{|\mathbf{q}|}\right)\right] \\
& T_{\mathbf{p}}^{\mathbf{k q}} \equiv \frac{|\mathbf{p}|}{\Omega_{0}^{\mathbf{q}} \Omega_{0}^{\mathbf{k}}} \frac{\Omega_{0}^{\mathbf{p}}}{\left(1-\beta_{0}^{\mathbf{p}} \Omega_{0}^{\mathbf{p}}\right)}\left[\frac{\left(\omega_{0}^{\mathbf{q}}\right)^{2}}{\omega_{0}^{\mathbf{p}} \omega_{0}^{\mathbf{k}}} \frac{|\mathbf{q}|^{2}}{|\mathbf{p}|^{2}} \beta_{0}^{\mathbf{q}}-\frac{\left(\omega_{0}^{\mathbf{k}}\right)^{2}}{\omega_{0}^{\mathbf{p}} \omega_{0}^{\mathbf{q}}} \frac{|\mathbf{k}|^{2}}{|\mathbf{p}|^{2}} \beta_{0}^{\mathbf{k}}\right] \\
& -\frac{|\mathbf{p}|}{2} \frac{\Omega_{0}^{\mathbf{p}}}{\omega_{0}^{\mathbf{p}}\left(1-\beta_{0}^{\mathbf{p}} \Omega_{0}^{\mathbf{p}}\right)}\left[\omega_{0}^{\mathbf{q}} \Omega_{0}^{\mathbf{q} *}\left(1-\frac{\omega_{0}^{\mathbf{q}} \Omega_{0}^{\mathbf{q}^{*}}}{\omega_{0}^{\mathbf{k}} \Omega_{0}^{\mathbf{k}}}\right)+\frac{\left(\omega_{0}^{\mathbf{k}} \Omega_{0}^{\mathbf{k}}-\omega_{0}^{\mathbf{q}} \Omega_{0}^{\mathbf{q}^{*}}\right)}{|\mathbf{p}|^{2} v_{3}^{\mathbf{k}} v_{3}^{\mathbf{q}^{*}}}\left(-\mathbf{q} \cdot v_{H}^{\mathbf{k}} v_{3}^{\mathbf{q}^{\prime *}}-\mathbf{q} \cdot v_{H}^{\mathbf{k}} \mathbf{k} \cdot v_{H}^{\mathbf{q} *}+|\mathbf{q}|^{2} v_{3}^{\mathbf{k}} v_{3}^{\mathbf{q}^{*}}+\mathbf{k} \cdot v_{H}^{\mathbf{q} \prime^{*}} v_{3}^{\mathbf{k}}\right)\right] \\
& -\frac{|\mathbf{p}|}{2} \frac{\Omega_{0}^{\mathbf{p}}}{\omega_{0}^{\mathbf{p}}\left(1-\beta_{0}^{\mathbf{p}} \Omega_{0}^{\mathbf{p}}\right)}\left[-\omega_{0}^{\mathbf{k}} \Omega_{0}^{\mathbf{k}}\left(1-\frac{\omega_{0}^{\mathbf{k}} \Omega_{0}^{\mathbf{k}}}{\omega_{0}^{\mathbf{q}} \Omega_{0}^{\mathbf{q}^{*}}}\right)+\frac{\left(\omega_{0}^{\mathbf{k}} \Omega_{0}^{\mathbf{k}}-\omega_{0}^{\mathbf{q}} \Omega_{0}^{\mathbf{q}^{*}}\right)}{|\mathbf{p}|^{2} v_{3}^{\mathbf{k}} v_{3}^{\mathbf{q}^{*}}}\left(-\mathbf{k} \cdot v_{H}^{\mathbf{q}^{*}} v_{3}^{\mathbf{k}^{\prime}}-\mathbf{k} \cdot v_{H}^{\mathbf{q} *} \mathbf{q} \cdot v_{H}^{\mathbf{k}^{*}}+|\mathbf{k}|^{2} v_{3}^{\mathbf{q}^{*}} v_{3}^{\mathbf{k}}+\mathbf{q} \cdot v_{H}^{\mathbf{k}^{\prime}} v_{3}^{\mathbf{q}^{*}}\right)\right] \\
& -\frac{|\mathbf{p}|}{2} \frac{\Omega_{0}^{\mathbf{p}}}{\omega_{0}^{\mathbf{p}}\left(1-\beta_{0}^{\mathbf{p}} \Omega_{0}^{\mathbf{p}}\right)}\left[\frac{\left(\omega_{0}^{\mathbf{k}}\right)^{2}}{\omega_{0}^{\mathbf{q}} \Omega_{0}^{\mathbf{q}^{*}}} \frac{1}{v_{3}^{\mathbf{k}}(0)}\left(\frac{\mathbf{q} \cdot v_{H}^{\mathbf{k}}}{|\mathbf{k}|}-\frac{v_{3}^{\mathbf{k}^{\prime}}}{|\mathbf{k}|}\right)-\frac{\left(\omega_{0}^{\mathbf{q}}\right)^{2}}{\omega_{0}^{\mathbf{k}} \Omega_{0}^{\mathbf{k}}} \frac{1}{v_{3}^{\mathbf{q}}(0)^{*}}\left(\frac{\mathbf{k} \cdot v_{H}^{\mathbf{q} *}}{|\mathbf{q}|}-\frac{v_{3}^{\mathbf{q}^{* *}}}{|\mathbf{q}|}\right)\right] \\
& T_{\mathbf{q}}^{\mathbf{p k}}=\frac{|\mathbf{q}|}{\Omega_{0}^{\mathbf{p}} \Omega_{0}^{\mathbf{k}}} \frac{\Omega_{0}^{\mathbf{q}}}{\left(1-\beta_{0}^{\mathbf{q}} \Omega_{0}^{\mathbf{q}}\right)}\left[\frac{\left(\omega_{0}^{\mathbf{p}}\right)^{2}}{\omega_{0}^{\mathbf{q}} \omega_{0}^{\mathbf{k}}} \frac{|\mathbf{p}|^{2}}{|\mathbf{q}|^{2}} \beta_{0}^{\mathbf{p}}-\frac{\left(\omega_{0}^{\mathbf{k}}\right)^{2}}{\omega_{0}^{\mathbf{q}} \omega_{0}^{\mathbf{p}}} \frac{|\mathbf{k}|^{2}}{|\mathbf{q}|^{2}} \beta_{0}^{\mathbf{k}}\right] \\
& -\frac{|\mathbf{q}|}{2} \frac{\Omega_{0}^{\mathbf{q}}}{\omega_{0}^{\mathbf{q}}\left(1-\beta_{0}^{\mathbf{q}} \Omega_{0}^{\mathbf{q}}\right)}\left[\omega_{0}^{\mathbf{p}} \Omega_{0}^{\mathbf{p} *}\left(1-\frac{\omega_{0}^{\mathbf{p}} \Omega_{0}^{\mathbf{p} *}}{\omega_{0}^{\mathbf{k}} \Omega_{0}^{\mathbf{k}}}\right)+\frac{\left(\omega_{0}^{\mathbf{k}} \Omega_{0}^{\mathbf{k}}-\omega_{0}^{\mathbf{p}} \Omega_{0}^{\mathbf{p} *}\right)}{|\mathbf{q}|^{2} v_{3}^{\mathbf{k}} v_{3}^{\mathbf{p}^{*}}}\left(-\mathbf{p} \cdot v_{H}^{\mathbf{k}} v_{3}^{\mathbf{p}^{\prime *}}-\mathbf{p} \cdot v_{H}^{\mathbf{k}} \mathbf{k} \cdot v_{H}^{\mathbf{p}^{*}}+v_{3}^{\mathbf{k}} v_{3}^{\mathbf{p}^{\prime \prime *}}+\mathbf{k} \cdot v_{H}^{\left.\mathbf{p}^{\prime *} * v_{3}^{\mathbf{k}}\right)}\right]\right. \\
& -\frac{|\mathbf{q}|}{2} \frac{\Omega_{0}^{\mathbf{q}}}{\omega_{0}^{\mathbf{q}}\left(1-\beta_{0}^{\mathbf{q}} \Omega_{0}^{\mathbf{q}}\right)}\left[-\omega_{0}^{\mathbf{k}} \Omega_{0}^{\mathbf{k}}\left(1-\frac{\omega_{0}^{\mathbf{k}} \Omega_{0}^{\mathbf{k}}}{\omega_{0}^{\mathbf{p}} \Omega_{0}^{\mathbf{p}^{*}}}\right)+\frac{\left(\omega_{0}^{\mathbf{k}} \Omega_{0}^{\mathbf{k}}-\omega_{0}^{\mathbf{p}} \Omega_{0}^{\mathbf{p}^{*}}\right)}{|\mathbf{q}|^{2} v_{3}^{\mathbf{k}} v_{3}^{\mathbf{p}^{*}}}\left(-\mathbf{k} \cdot v_{H}^{\mathbf{p}^{*}} v_{3}^{\mathbf{k}^{\prime}}-\mathbf{k} \cdot v_{H}^{\mathbf{p} *} \mathbf{p} \cdot v_{H}^{\mathbf{k}^{*}}+v_{3}^{\mathbf{p}^{*}} v_{3}^{\mathbf{k}^{\prime \prime}}+\mathbf{p} \cdot v_{H}^{\mathbf{k}^{\prime}} v_{3}^{\mathbf{p}^{*}}\right)\right] \\
& -\frac{|\mathbf{q}|}{2} \frac{\Omega_{0}^{\mathbf{q}}}{\omega_{0}^{\mathbf{q}}\left(1-\beta_{0}^{\mathbf{q}} \Omega_{0}^{\mathbf{q}}\right)}\left[\frac{\left(\omega_{0}^{\mathbf{k}}\right)^{2}}{\omega_{0}^{\mathbf{p}} \Omega_{0}^{\mathbf{p}^{*}}} \frac{1}{v_{3}^{\mathbf{k}}(0)}\left(\frac{\mathbf{p} \cdot v_{H}^{\mathbf{k}}}{|\mathbf{k}|}-\frac{v_{3}^{\mathbf{k}^{\prime}}}{|\mathbf{k}|}\right)-\frac{\left(\omega_{0}^{\mathbf{p}}\right)^{2}}{\omega_{0}^{\mathbf{k}} \Omega_{0}^{\mathbf{k}}} \frac{1}{v_{3}^{\mathbf{p}}(0)^{*}}\left(\frac{\mathbf{k} \cdot v_{H}^{\mathbf{p} *}}{|\mathbf{p}|}-\frac{v_{3}^{\mathbf{p}^{* *}}}{|\mathbf{p}|}\right)\right] \text {. }
\end{aligned}
$$

Here we introduced auxiliary notation, denoting the zero-current frequency $\omega_{0}^{\mathbf{k}}=\sqrt{g \mid \mathbf{k}}$, a Doppler-shifted frequency $\Omega_{\mathbf{k}}(z)=\omega_{\mathbf{k}}-\mathbf{k} \cdot \mathbf{U}(z)$, the dimensionless ratio $\Omega_{0}^{\mathbf{k}}=\Omega_{\mathbf{k}}(0) / \omega_{0}^{\mathbf{k}}$, a dimensionless measure of shear strength $\beta_{0}^{\mathbf{k}}=$ $\mathbf{k} \cdot \mathbf{U}^{\prime}(0) / 2|\mathbf{k}| \omega_{0}^{\mathbf{k}}$, and the 2-dimensional horizontal velocity amplitude $v_{H}(z)=\left(v_{1}(z), v_{2}(z)\right)$ where $v_{1}$ and $v_{2}$ are defined by Eq. (13). All terms above are evaluated at the mean surface height $z=0$ and ${ }^{*}$ denotes complex conjugation. 
[1] Peregrine, D. H. and Thomas, G. P. “Finite-amplitude deep-water waves on currents.” Phil. Trans. R. Soc. Lond. A 292, 371-390 (1979).

[2] Lillibridge, J. L., Hitchcock, G., Rossby, T., Lessard, E., Mork M. and Golmen, L. 'Entrainment and Mixing of Shelf/Slope Waters in the Near-Surface Gulf Stream.” J. Geo. Res. 95, no. c8, pp. 13,065-13,087 (1990)

[3] Dalrymple, R. “A finite amplitude wave on a linear shear current.” J. of Geo, Res. 79, Issue 30, pages 4498-4504, (1974)

[4] Tamura, H., Waseda, T., Miyazawsa, Y., and komatsu, K. "Current-induced modulation of the ocean wave spectrum and the role of nonlinear energy transfer:” J. Phys. Oceanogr. 38, 2662-2684 (2008).

[5] Slunyaev ,A., Didenkulova, I. and Pelinovsky, E. “Rogue Waters.” Contemporary Physics 52, Issue 6, 571-590 (2011).

[6] Pierson W., and Moskowitz, L. "A proposed spectral form for fully developed wind seas based on the similarity theory of s.a. kitaigorodskii." J. Geophys. Res. 69, 51815190 (1964).

[7] Donelan, M., Hamilton, J., and Hui, W. "Directional spectra of wind generated waves.” Phil. Trans. R. Soc. London, A, 315, 509562 (1985).

[8] Elfouhaily, T., Chapron, B., Katsaros, K., and Vandemark, D. "A unified directional spectrum for long and short wind-driven waves." J. Geophys. Res., 102, 15,78115,796 (1997).

[9] Longuet-Higgins, M.S., and Stewart, R.W. “The changes in amplitude of short gravity waves on steady non-uniform currents.” J. Fluid Mech. 10, 529-549 (1961).

[10] Craik, A.D.D. “Resonant gravity-wave interactions in a shear flow.” J. Fluid Mech. 34, 531-549 (1968).

[11] Phillips, O.M. “On the dynamics of unsteady gravity waves of finite amplitude. Part 1. The elementary interactions.” J. Fluid Mech. 9 (2), 193 (1960).

[12] Longuet-Higgins, M.S. "Resonant interactions between two trains of gravity waves.” J. Fluid Mech. 12 (3), 321 (1962).

[13] Dyachenko, A.I. and Zakharov, V.E. “Is free-surface hydrodynamics an integrable system?” Phys. Lett. A 190 (2), 144-148 (1994).

[14] Oliveras, K., and Vasan, V. “A new equation describing traveling water waves.” J. Fluid Mech. 773, 514-522 (2013).

[15] Hur, V. M., and Lin, Z. “Unstable surface waves in running water.” Commun. Math. Phys. 282, no. 3, 733-796, (2008).

[16] Koslov, V and Kuznetzov, N. "Dispersion equation for water waves with vorticity and Stokes waves on flow with counter-currents." preprint arXiv:1207.5181 (2013).

[17] Wolfram Research, Inc., Mathematica Version 10.0, Champaign, IL (2014)

[18] Choi, W., "Nonlinear surface waves interacting with a linear shear current." Math. and Comp. in Sim. 80 29-36 (2009).

[19] McHugh, J.P. "Surface waves on an inviscid shear flow in a channel." Wave Motion 19, 135-144 (1994).

[20] Nwogu, O. "Interaction of finite-amplitude waves with vertically sheared current fields.” J. Fluid Mech. 627 179-213 (2009)

[21] Zakharov, V.E., and Shrira, V.I. “On the formation of the directional spectra of wind waves.” Sov. Phys. JETP 71, 1091-1100 (1990).

[22] Carpenter, J.R., Tedford, E.W., Heifetz, E., and Lawrence, G.A. "Instability in stratified shear flow: Review of a physical interpretation based on interacting waves." Ap. Mech. Rev. 64, 060801 (2013).

[23] Shrira, V.I. "Surface waves on shear currents: Solution of the boundary-value problem.” J. Fluid Mech. 253, 565-584 (1993).

[24] Voronovich, A.G., Lobanov, E.D., and Ryback, S.A. "On the stability of gravitational-capillary waves in the presence of a vertically nonuniform current.” Izv. Atmos. Ocean Phys. 26, 220222 (1980).

[25] Bakas, N.A., and Ioannou, P.J. "Modal and nonmodal growths of inviscid planar perturbations in shear flows with a free surface." Phys. Fluids 21, 024102 (2009).

[26] Ball, F.K. "Energy transfer between external and internal gravity waves.” J. Fluid Mech. 19 (3), 465 (1964)

[27] Joyce, T.M. “Nonlinear interactions among standing surface and internal gravity waves.” J. Fluid Mech. 63 (4), $801-825$ (1974).

[28] Alam, M.-R. “A new triad resonance between co-propagating surface and interfacial waves.” J. Fluid Mech. 691, 267-278 (2012).

[29] Craig, W., Guyenne, P., and Sulem, C. “Coupling between internal and surface waves.” Natural Hazards 57 (3), $617-642$ (2010).

[30] Drazin, P.G. and Reid, W.H., "Hydrodynamic Stability.” Cambridge University Press (2004).

[31] Shrira, V., and Sazonov, I., "Quasi-modes in boundary-layer-type shear flows. Part 1. Inviscid two-dimensional spatially harmonic perturbations.”, J. of Fluid Mech. 446, 133-171 (2001).

[32] Chen, R.M.m and Walsh, S. "Continuous dependence on the density for stratified steady water waves.” arXiv:1408.5030.

[33] Wunsch, S., and Keller, K. “Unstable modes of a sheared pycnocline above a stratified layer.” Dyn. Atmos. Oceans 60, 1-27 (2013).

[34] Rudin, W. "Principles of Mathematical Analysis.” Int. Series in Pure Applied Mathematics (1976).

[35] Majda, Andrew J., and Pedro Embid. "Averaging over fast gravity waves for geophysical flows with unbalanced initial data." Theoretical and computational fluid dynamics 11, no. 3-4 (1998)

[36] Alam, M.-R., Liu, Y., and Yu, D.K.P. “Attenuation of short surface waves by the sea floor via nonlinear sub-harmonic interaction.” J. Fluid Mech. 689, 529-540 (2011).

[37] Alam, M.-R., Liu, Y., and Yu, D.K.P. “Attenuation of short surface waves by the sea floor via nonlinear sub-harmonic interaction.” J. Fluid Mech. 689, 529-540 (2011).

[38] Alam, M.-R., Liu, Y., and Yu, D.K.P. "Resonant wave signature of an oscillating and translating disturbance in a two-layer density stratified fluid.” J. Fluid Mech. 675, 477-494 (2011).

[39] Alam, M.-R., Liu, Y., and Yu, D.K.P. “Oblique sub- and super-harmonic Bragg resonance of surface waves by bottom ripples.” J. Fluid Mech. 643, 437-447 (2010)

[40] Alam, M.-R., Liu, Y., and Yu, D.K.P. “Bragg resonance of waves in a two-layer fluid propagating over bottom ripples. Part I. Perturbation analysis.” J. Fluid Mech. 624, 191-224 (2009).

[41] Kaihatu, J.M., and Tahvildari, N. "The combined effect of wave-current interaction and mud-induced damping on nonlinear wave evolution." Ocean Modelling (2012)

[42] Plougonven, R., and J. Vanneste. “Quasigeostrophic dynamics of a finite-thickness tropopause.” Journal of the Atmospheric Sciences 67.10 (2010): 3149-3163.

[43] Kent, C.P., and Choi, W. “An explicit formulation for the evolution of nonlinear surface waves interacting with a submerged body.” Int. J. Numer. Meth. Fluids 55, 1019-1038 (2007). 